KANAZAWA-12-08

March, 2012

\title{
Super Flavorsymmetry with Multiple Higgs Doublets*
}

\author{
Jisuke $\mathrm{Kubo}^{\dagger}$ \\ Institute for Theoretical Physics, Kanazawa University, \\ Kanazawa 920-1192, Japan
}

\begin{abstract}
The meaning of non-abelian super flavorsymmetry in the presence of Multiple Higgs Doublets is twofold: The first one is to order the Yukawa sector of the standard model, while generating the Cheng-Sher suppression of tree-level Flavor Changing Neutral Currents (FCNCs), and the second one is to soften the fine tuning problem in the scalar potential and at the same time to suppress FCNCs coming from the soft breaking of supersymmetry. If spontaneous $\mathrm{CP}$ violation is combined with non-abelian super flavorsymmetry the $\mathrm{CP}$ phases in the soft supersymmetry breaking sector can disappear. This feature of super flavorsymmetry is reviewed and elaborated in a supersymmetric model of flavor based on the finite group $Q_{6}$. The predictions of the model in ferimion mass and mixing, and flavor and $\mathrm{CP}$ violation can be tested in future experiments.
\end{abstract}

\footnotetext{
* submitted to Fortschritte der Physik (April 2012)

$\dagger$ e-mail: jik@hep.s.kanazawa-u.ac.jp
} 


\section{INTRODUCTION}

Introducing flavor symmetry based on a non-abelian finite group into the standard model (SM) means going beyond the SM [1]. The main reason of the introduction of flavor symmetry lies in the Yukawa sector of the SM, because the most of the free parameters of the SM are involved in this sector, and there exists within the SM no principle how to fix its structure. There are many guidelines to go beyond the SM. Therefore, depending on the guideline, how to introduce non-abelian flavor symmetry is different [2]-[17]. The difference of the guidelines may be summarized as:

1. How it is broken.

Since there exists no exact non-abelian flavor symmetry in the SM, it hast to be broken: It is spontaneously, softly or hardly broken.

2. The scale of its breaking.

It is a low energy scale or a much higher scale, e.g., a GUT scale.

3. Renormalizability.

The extended model is renormalizable or non-renormalizable.

4. On which sector it is effectively acting: On the quark sector; on the lepton sector; or on both the quark and the lepton sector.

In addition to the above four categories we can add whether the model is supersymmetric or not, it is a four dimensional model or not, and whether CP is spontaneously, softly or explicitly broken. Note that no gauge group and no flavor group are fixed yet. However, except for the existence of dark matter and the neutrino mixing and masses, despite the great efforts of experimentalists, there is no experimental hint how to go beyond the SM.

Under these circumstances I prefer to attach importance to the experimental verifiability of a model. It is clear that the lower the scale of the breaking of flavor symmetry is, the more verifiable is the model. Moreover, if flavor symmetry is hardly broken, it is not testable in a strict sense. Further, to decide whether the model should be renormalizable or not, I would like to follow the attempt of Kobayashi and Maskawa [18], who introduced CP violation within the renormalizable extension of the two generation SM. From these considerations I decided to restrict myself, in this contribution to the special issue, to renormalizable models of flavor, where non-abelian discrete flavor symmetries are at most softly broken at low energy. One immediately realizes that it is impossible to present a realistic model along this line, unless one introduces multiple Higgs doublets which belong to non-trivial representations of the corresponding non-abelian finite group. Therefore, flavor symmetry is at least spontaneously broken at the electroweak scale together with the electroweak gauge symmetry. This can lead to severe problems with Flavor Changing Neutral Currents (FCNCs), if the extra Higgs bosons are not heavy enough [19]. I will not discuss the details of the different constraints such as $\mu \rightarrow 3 e$ coming from flavor violations (see [20] for instance). I simply assume that their masses are larger than several $\mathrm{TeV}$ and the electroweak precision constraints are satisfied [21].

In contrast to two Higgs doublet models without non-abelian flavor symmetry[22], the size of the Yukawa couplings are not arbitrary, because fermion masse and mixing would not come out right. However, the scale is not $O(100) \mathrm{TeV}$ [19], because the Cheng-Sher mechanism [23], which suppresses the tree-level FCNCs, is partially working thanks to flavor 
symmetry and making the scale of flavor symmetry decrease down to tera scale. The presence of extra heavy Higgs doublet bosons of tera scale causes another problem, a fine tuning in the scalar potential. The fine tuning can be made stable against radiative corrections by supersymmetry [24 26]. This is why I will concentrate on supersymmetric models of flavor. Due to the very nature of non-renormalization theorem, extended to softly broken supersymmetric theories [27, 28], the soft breaking terms of flavor symmetry can be classified, in addition to according to their canonical dimensions (Symanzik's theorem [29]). I will discuss this matter in a general manner in Sect. 2 and 3.

The meaning of non-abelian flavor symmetry in supersymmetric models is twofold: The first one is to introduce a principle in the Yukawa sector as it is the case for nonsupersymmetric models, while generating the Cheng-Sher suppression, and the second one is to suppress FCNCs coming from the soft breaking of supersymmetry [30]-[35], while supersymmetry softens the fine tuning problem in the scalar potential. This mechanism to suppress FCNCs is alternative to hidden sector supersymmetry breaking. (The both mechanisms can be of course combined.) The suppression mechanism has been observed in many concrete models [7, 11, 36]-[46], and it is in fact very general. In certain models, like the one [7] which I will discuss in detail, the $\mathrm{CP}$ violating phases in the soft supersymmetry breaking (SSB) sector disappear, if CP is spontaneously broken: A phase self-alignment works [7].

From Sect. 4 on I will discuss the specific supersymmetric flavor model based on the finite flavor group $Q_{6}$ [7, 11], [43]- [46]. This model will illustrate the basic features of any

realistic supersymmetric flavor models with multiple Higgs doublet supermultiplets [47]. The predictions on fermion mass and mixing are discussed in Sect. 5, and I will show in Sect. 6 how FCNCs and CP violations coming from the supersymmetry breaking are suppressed by $Q_{6}$ flavor symmetry. Sect. 7 is devoted to Summary.

\section{FLAVOR SYMMETRY AND NON-RENORMALIZATION THEOREM IN SOFTLY BROKEN SUPERSYMMETRIC THEORIES}

Favor symmetry can control the structure of the independent parameters of a theory. In supersymmetric theories, moreover, the non-renormalization theorem allows to drop certain couplings and also to relate them with each other, without facing contradictions with renormalizability. We would like to discuss the role of the non-renormalization theorem for flavor symmetry and vice versa. We begin by considering a generic $N=1$ supersymmetric gauge theory whose superpotential is given by

$$
W(\Phi)=\frac{1}{6} Y^{i j k} \Phi_{i} \Phi_{j} \Phi_{k}+\frac{1}{2} \mu^{i j} \Phi_{i} \Phi_{j} .
$$

The soft supersymmetry breaking (SSB) Lagrangian is given by

$$
\begin{aligned}
L(\Phi, W)= & -\left(\int d^{2} \theta \eta\left(\frac{1}{6} h^{i j k} \Phi_{i} \Phi_{j} \Phi_{k}+\frac{1}{2} b^{i j} \Phi_{i} \Phi_{j}+\frac{1}{2} M_{g} W_{A}^{\alpha} W_{A \alpha}\right)+\text { h.c. }\right) \\
& -\int d^{4} \theta \tilde{\eta} \eta \overline{\Phi^{j}}\left(m^{2}\right)_{j}^{i}\left(e^{2 g V}\right)_{i}^{k} \Phi_{k}
\end{aligned}
$$

where $\eta=\theta^{2}, \tilde{\eta}=\tilde{\theta}^{2}$ are the external spurion superfields and $M_{g}$ is the gaugino mass.

To proceed we take into account the presence of flavor symmetry. We recall that the Dterms are renormalized and the wave function renormalization can mix matter superfields $\Phi_{i}$ 
in general. If a non-diagonal (infinite) kinetic term is induced, a corresponding non-diagonal counter term should be added. In what follows we assume that the kinetic mixing among $\Phi_{i}$ 's is forbidden by flavor symmetry. This implies that the anomalous dimensions $\gamma^{i}$ are diagonal, i.e., $\gamma^{i}{ }_{j}=\delta^{i}{ }_{j} \gamma_{j}$. Then the $\beta$ functions of $Y, \mu, h$ and $m^{2}$ are given by [28, 48]

$$
\begin{aligned}
\beta_{Y}^{i j k} & =Y^{i j k}\left(\gamma_{i}+\gamma_{j}+\gamma_{k}\right), \beta_{\mu}^{i j}=\mu^{i j}\left(\gamma_{i}+\gamma_{j}\right) \\
\beta_{h}^{i j k} & =\left(h^{i j k}-2 Y^{i j k} \mathcal{O}\right)\left(\gamma_{i}+\gamma_{j}+\gamma_{k}\right), \beta_{b}^{i j}=\left(b^{i j}-2 \mu^{i j} \mathcal{O}\right)\left(\gamma_{i}+\gamma_{j}\right), \\
\left(\beta_{m^{2}}\right)_{l} & =\left[\Delta+X \frac{\partial}{\partial g}\right] \gamma_{l}, \mathcal{O}=\left(M_{g} g^{2} \frac{\partial}{\partial g^{2}}-h^{l m n} \frac{\partial}{\partial Y^{l m n}}\right) \\
\Delta & =2 \mathcal{O O} \mathcal{O}^{*}+2\left|M_{g}\right|^{2} g^{2} \frac{\partial}{\partial g^{2}}+\tilde{Y}_{l m n} \frac{\partial}{\partial Y_{l m n}}+\tilde{Y}^{l m n} \frac{\partial}{\partial Y^{l m n}}
\end{aligned}
$$

where $\left(\gamma_{1}\right)_{i}=\mathcal{O} \gamma_{i}, \quad Y_{l m n}=\left(Y^{l m n}\right)^{*}$, and $\tilde{Y}^{i j k}=Y^{i j k}\left(m_{i}^{2}+m_{j}^{2}+m_{k}^{2}\right), X=$ $\frac{-\left|M_{g}\right|^{2} C(G)+\sum_{l} m_{l}^{2} T\left(R_{l}\right)}{C(G)-8 \pi^{2} / g^{2}}$, and $T\left(R_{l}\right)$ is the Dynkin index of $R_{l}$, and $C_{2}(G)$ is the quadratic Casimir of the adjoint representation of the gauge group $G$. From these equations we may conclude (which is basically Symanzik's theorem [29] applied to softly broken supersymmetric gauge theories):

Th1. The $\mu$ sector can have a flavor symmetry which is different from the flavor symmetry of the Yukawa sector if both symmetries are compatible with respect to renormalization of $\mu^{i j}$. That is, if $\mu^{i j}\left(\gamma_{i}+\gamma_{j}\right) \sim \mu^{i j}$.

Th2. It is consistent to introduce into the soft tri-linear couplings the same flavor symmetry as that of the Yukawa couplings, even if it is violated in other sectors.

Th3. The flavor symmetry which protects the kinetic mixing among $\Phi_{i}$ 's ensures that $\left(m^{2}\right)_{j}^{i}$ are diagonal. If the Yukawa couplings and soft tri-linear couplings have the flavor symmetry, the soft scalar mass terms, too, can have the flavor symmetry, even if the $\mu$ and $b$ terms do not respect the flavor symmetry.

Th4. The $b$ terms associated with the $\mu$ terms should always exist (see (5)). But the $b$ sector has no influence on the infinite renormalization of the parameters in other sectors. So the violation of a symmetry in the $b$ sector is doubly soft. Similarly, the soft scalar mass terms and soft tri-linear couplings have no influence on the infinite renormalization of the $b$ terms.

\section{SOFTLY BROKEN FLAVOR SYMMETRIES AND SUPERSYMMETRY}

We restrict ourselves to the gauge group $S U(3)_{C} \times S U(2)_{L} \times U(1)_{Y}$ and require renormalizability as it is announced. As in the non-supersymmetric case, realistic softly broken supersymmetric models with a low energy flavor symmetry will contain certain number of Higgs doublet supermultiplets pairs $\Phi_{I}^{u}$ and $\Phi_{I}^{d}$. The superpotential in the matter sector is given by $W_{Q}+W_{L}+W_{M}$, where

$$
\begin{aligned}
W_{Q} & =Y_{i j}^{u I} Q_{i} U_{j}^{c} \Phi_{I}^{u}+Y_{i j}^{d I} Q_{i} D_{j}^{c} \Phi_{I}^{d}, W_{L}=Y_{i j}^{e I} L_{i} E_{j}^{c} \Phi_{I}^{d}+Y_{i j}^{\nu I} L_{i} N_{j}^{c} \Phi_{I}^{u}, \\
W_{M} & =\frac{1}{2} M_{i j} N_{i}^{c} N_{j}^{c},
\end{aligned}
$$

where $Q$ and $L$ stand for $S U(2)_{L}$ doublets of the quark and lepton supermultiplets, respectively, where $i$ and $j$ are flavor group indices. Similarly, $U^{c}, E^{c}$ and $N^{c}$ stand for $S U(2)_{L}$ 
singlets of the quark, charged lepton and right-handed neutrino supermultiplets. In the following discussions we denote the scalar and fermionic components of the supermultiplets as

$$
Q=\left(\tilde{q}_{L}, q_{L}\right)=\left(\begin{array}{cc}
\tilde{u}_{L}, & u_{L} \\
\tilde{d}_{L}, & d_{L}
\end{array}\right), U^{c}=\left(\tilde{u}_{R}^{*}, u_{R}^{c}\right)
$$

and similarly for $D^{c}, L$, etc. The Higgs supermultiplets are

$$
\Phi^{u}=\left(h^{u}, \tilde{h}^{u}\right)=\left(\begin{array}{cc}
h^{u+}, & \tilde{h}^{u+} \\
h^{u 0}, & \tilde{h}^{u 0}
\end{array}\right), \Phi^{d}=\left(h^{d}, \tilde{h}^{d}\right)=\left(\begin{array}{cc}
h^{d 0}, & \tilde{h}^{d 0} \\
h^{d-} & \tilde{h}^{d-}
\end{array}\right)
$$

and we assume that the neutral components of the Higgs fields, $h^{u 0}$ and $h^{d 0}$, acquire a (complex) VEV.

The $\mu$ terms: The most general, renormalizable, Higgs superpotential $W_{H}$ has the form

$$
W_{H}=\sum_{I, J} \mu_{I J} \Phi_{I}^{u} \Phi_{J}^{d}
$$

The superpotential $W_{H}$ often has a symmetry larger, quite often continuos symmetry, than the one in the Yukawa sector. The large continues symmetry has to be explicitly broken in the scalar potential to avoid the appearance of the massless NG bosons and fermions. Another problem is that certain fermionic components of $\Phi_{I}$ are massless. The most general form of the mass matrix $\mathbf{M}_{N}^{F}$ of the neutralinos in the prepense of $N$ pairs of the Higgs doublet supermultiplets can be written as

$$
\mathbf{M}_{N}^{F}=\left(\begin{array}{cccccc}
\mathbf{M}_{g g} & \mathbf{M}_{g 1} & \mathbf{M}_{g 2} & \mathbf{M}_{g 3} & \cdots & \mathbf{M}_{g N} \\
\mathbf{M}_{g 1}^{T} & \mathbf{M}_{11} & \mathbf{M}_{12} & \mathbf{M}_{13} & \cdots & \mathbf{M}_{1 N} \\
\mathbf{M}_{g 2}^{T} & \mathbf{M}_{12}^{T} & \mathbf{M}_{22} & \mathbf{M}_{23} & \cdots & \mathbf{M}_{2 N} \\
\mathbf{M}_{g 3}^{T} & \mathbf{M}_{13}^{T} & \mathbf{M}_{23}^{T} & \mathbf{M}_{33} & \cdots & \mathbf{M}_{3 N} \\
. & . . & . . & . . & \\
\mathbf{M}_{g N}^{T} & \cdots & \cdots & \cdots & \cdots & \mathbf{M}_{N N}
\end{array}\right)
$$

where

$$
\mathbf{M}_{g g}=\left(\begin{array}{cc}
M_{1} & 0 \\
0 & M_{2}
\end{array}\right), \mathbf{M}_{g I}=\left(\begin{array}{cc}
s_{W}\left(v_{I}^{u} / v\right) M_{Z} & -s_{W}\left(v_{I}^{d} / v\right) M_{Z} \\
-c_{W}\left(v_{I}^{u} / v\right) M_{Z} & c_{W}\left(v_{I}^{u} / v\right) M_{Z}
\end{array}\right), \mathbf{M}_{I J}=\left(\begin{array}{cc}
0 & -\mu_{I J} \\
-\mu_{I J} & 0
\end{array}\right)
$$

$s(c)_{W}=\sin (\cos ) \theta_{W}$, and $M_{1,2}$ are gaugino masses. Similarly, the mass matrix of the charginos $\mathbf{M}_{C}$ has the form

$$
\mathbf{M}_{C}^{F}=\left(\begin{array}{cccc}
M_{2} & \ldots & \left(v_{J}^{d} / v\right) M_{W} & \ldots \\
\vdots & & \vdots & \\
\left(v_{I}^{u} / v\right) M_{W} & \ldots & \mu_{I J} & \ldots \\
\vdots & & \vdots &
\end{array}\right)
$$

To give the massless fermions a mass we can break flavor symmetry at the superpotential level, which however should be consistent with Th1.

The soft scalar mass terms: Since flavor symmetry for the soft scalar mass terms of the matter fields plays a crucial role to suppress FCNCs in the SSB sector, we assume that 
flavor symmetry is not broken by the soft scalar mass terms. This requires implicitly that flavor symmetry is respected by the soft tri-linear couplings, because the breaking by the soft tri-linear couplings is not consistent with the assumption that flavor symmetry is respected by the soft scalar mass terms. The most general form of the soft scalar mass terms for the multi Higgs doublet fields is

$$
\mathcal{L}_{m_{H}^{2}}=\sum_{I}\left(m_{H_{I}^{u}}^{2}\left(h_{I}^{u}\right)^{*} h_{I}^{u}+m_{H_{I}^{d}}^{2}\left(h_{I}^{d}\right)^{*} h_{I}^{d}+\right)+\sum_{I<J}\left(m_{H_{I J}^{u}}^{2}\left(h_{I}^{u}\right)^{*} h_{J}^{u}+m_{H_{I J}^{d}}^{2}\left(h_{I}^{d}\right)^{*} h_{J}^{d}+h . c .\right) .
$$

The superpotential (7) gives rise to quadratic terms, too:

$$
\mathcal{L}_{\mu^{2}}=\sum_{I, J, K}\left(\mu_{I K}^{*} \mu_{J K}\left(h_{I}^{u}\right)^{*} h_{J}^{u}+\mu_{K I}^{*} \mu_{K J}\left(h_{I}^{d}\right)^{*} h_{J}^{d}\right)
$$

The $b$ terms:

The $b$ sector should contain at least terms which correspond to the $\mu$ terms in $W_{H}$ :

$$
\mathcal{L}_{b}=\sum_{I J}\left(b_{I J} h_{I}^{u} h_{J}^{d}+\text { h.c. }\right)
$$

If flavor symmetry should be exact, then $b_{I J}$ in (11) have the same structure as $\mu_{I J}$ in (11). As we discussed, the breaking of flavor symmetry by the $b$ terms is doubly soft; it is soft, because the canonical dimension of the breaking operators is two, and the breaking has no influence on the infinite renormalization of the soft scalar masses (see Th2). Therefore, if the Higgs superpotential $W_{H}$ given in (11) has an accidental continuous symmetry, one can break it softly by the $b$ terms to avoid the appearance of the NG bosons and fermions.

If the $b$ parameters are complex, $\mathrm{CP}$ is explicitly broken by the $b$ terms; the breaking is doubly soft. CP breaking by the $b$ terms is an economic way, because one can overcome the domain wall problem which might appear when discrete flavor symmetries are spontaneously broken, and one may moreover obtain extra CP phases to make electroweak baryogenesis possible.

Softly broken CP invariance: In the presence of Higgs doublet pairs only, the quartic terms in the Higgs scalar potential come only from the D-terms, which are positive semi definite. Therefore, to show that the potential minimum is not at the origin, one needs to consider only the quadratic terms

$$
\mathcal{L}_{m_{H}^{2}}+\mathcal{L}_{b}=\frac{1}{2} \sum_{I, J}\left(\begin{array}{c}
\varphi_{I}^{u} \\
\chi_{I}^{u} \\
\varphi_{I}^{d} \\
\chi_{I}^{d}
\end{array}\right)^{T} \quad\left(\mathbf{M}_{\mathbf{H}}^{2}\right)_{I J}\left(\begin{array}{c}
\varphi_{J}^{u} \\
\chi_{J}^{u} \\
\varphi_{J}^{d} \\
\chi_{J}^{d}
\end{array}\right)
$$

where $h_{I}^{u, d}=\left(\varphi_{I}^{u, d}+i \chi_{I}^{u, d}\right) / \sqrt{2}$, and the $4 \times 4$ matrix $\left(\mathbf{M}_{\mathbf{H}}^{2}\right)_{I J}$ has the form

$$
\left(\mathbf{M}_{\mathbf{H}}^{\mathbf{2}}\right)_{I J}=\left(\begin{array}{cccc}
m_{H_{I J}^{u}}^{2}+\mu_{I K}^{*} \mu_{J K} & m_{H_{I J}^{u}}^{2}+\mu_{I K}^{*} \mu_{J K} & \operatorname{Re}\left(b_{I J}\right) & -\operatorname{Im}\left(b_{I J}\right) \\
m_{H_{I J}^{u}}^{2}+\mu_{I K}^{*} \mu_{J K} & m_{H_{I J}^{u}}^{2}+\mu_{I K}^{*} \mu_{J K} & -\operatorname{Im}\left(b_{I J}\right) & -\operatorname{Re}\left(b_{I J}\right) \\
\operatorname{Re}\left(b_{J I}\right) & -\operatorname{Im}\left(b_{J I}\right) & m_{H_{I J}^{d}}^{2}+\mu_{K I}^{*} \mu_{K J} & m_{H_{I J}^{d}}^{2}+\mu_{K I}^{*} \mu_{K J} \\
-\operatorname{Im}\left(b_{J I}\right) & -\operatorname{Re}\left(b_{J I}\right) & m_{H_{I J}^{d}}^{2}+\mu_{K I}^{*} \mu_{K J} & m_{H_{I J}^{d}}^{2}+\mu_{K I}^{*} \mu_{K J}
\end{array}\right) .
$$


The relevant matrix is the $4 N \times 4 N$ matrix $\mathcal{M}_{H}^{2}(N$ is the number of the Higgs doublet pairs)

$$
\mathcal{M}_{H}^{2}=\left(\begin{array}{ccc}
\left(\mathbf{M}_{\mathbf{H}}^{\mathbf{2}}\right)_{11} & \left(\mathbf{M}_{\mathbf{H}}^{\mathbf{2}}\right)_{11} & \ldots \\
\left(\mathbf{M}_{\mathbf{H}}^{\mathbf{2}}\right)_{21} & \left(\mathbf{M}_{\mathbf{H}}^{\mathbf{2}}\right)_{22} & \ldots \\
\ldots & \ldots & \ldots
\end{array}\right)
$$

whose eigenvalues control the behavior of the Higgs scalar potential in the $4 N$ dimensional space. We find that all the eigenvalues of $\mathcal{M}_{H}^{2}$ are doubly degenerate, and that two orthogonal eigenvectors of the same eigenvalue can be always written in the form $\vec{u}_{A}=\left(u_{1}, u_{2}, u_{3}, u_{4}, \ldots\right)$ and $\vec{u}_{B}=\left(u_{2},-u_{1},-u_{4}, u_{3}, \ldots\right)$. This is due to the $U(1)_{Y}$ gauge invariance: All the directions defined by a linear combination of $\vec{u}_{A}$ and $\vec{u}_{B}$ are physically equivalent. That is, one can make an appropriate linear combination such that at least one imaginary part vanishes. If on the other hand all the imaginary parts of $m_{H}^{2}$ 's and $b$ 's vanish, then the eigenvectors can always be written as $\left(c_{\theta} u_{1},-s_{\theta} u_{1}, c_{\theta} u_{3}, s_{\theta} u_{3}, c_{\theta} u_{5},-s_{\theta} u_{5}, \ldots\right)$, where $c_{\theta}=\cos \theta$ and $s_{\theta}=\sin \theta$, and the angle $\theta$ is, thanks to $U(1)_{Y}$, a free-choosable parameter. Therefore, we can always choose $\theta$ to be zero, i.e., all the imaginary parts vanish in this direction, implying that spontaneous $\mathrm{CP}$ violation at the tree level is not possible [49]. Therefore, at least some of $m_{H}^{2}$ or/and of $b$ should be complex so that the Higgs doublet fields can acquire imaginary VEV. Needless to say that negative eigenvalues of (20) have to exist for the potential minimum to be located other than at the origin.

The tri-linear couplings : The soft tri-linear couplings corresponding to the superpotential (7) is

$$
\mathcal{L}_{A}=h_{i j}^{u I} \tilde{q}_{L i} \tilde{u}_{R j}^{*} h_{I}^{u}+h_{i j}^{d I} \tilde{q}_{L i} \tilde{u}_{R j}^{*} h_{I}^{d}+h_{i j}^{e I} \tilde{l}_{L i} \tilde{e}_{R j}^{*} h_{I}^{d}+h_{i j}^{\nu I} \tilde{l}_{L i} \tilde{\nu}_{R j}^{*} h_{I}^{u}+h . c . .
$$

According to Th2 and Th3, the soft tri-linear couplings and soft scalar mass terms can have the same flavor symmetry as that of the Yukawa sector, even if flavor symmetry is softly broken in the $\mu$ sector and by the $b$ terms. Further, the superpotential (7) gives rise to tri-linear couplings, too:

$\mathcal{L}_{\mu}=\mu_{I J}\left(Y_{i j}^{d J}\right)^{*} \tilde{Q}_{i}^{\dagger} \tilde{d}_{R j} h_{I}^{u}-\mu_{I J}\left(Y_{i j}^{u I}\right)^{*} \tilde{Q}_{i}^{\dagger} \tilde{u}_{R j} h_{J}^{d}+\mu_{I J}\left(Y_{i j}^{e J}\right)^{*} \tilde{L}_{i}^{\dagger} \tilde{e}_{R j} h_{I}^{u}-\mu_{I J}\left(Y_{i j}^{\nu I}\right)^{*} \tilde{L}_{i}^{\dagger} \tilde{n}_{R j} h_{J}^{d}+h . c .$,

which contributes to the soft left-right mass matrices of the sfermions as we will see later on.

\section{A CONCRETE MODEL OF FLAVOR BASED ON THE FINITE GROUP $Q_{6}$ [7]}

In Table 1 the $Q_{6}$ assignment of the quark, lepton and Higgs chiral supermultiplets is given ${ }^{1}$. In addition to $Q_{6}$ flavor symmetry, a flavor universal $Z_{4}$ symmetry is introduced. Owing to this $Z_{4}$, even after spontaneous symmetry breaking, unbroken interchange symmetries $\mathcal{P}_{I, I I}$ survive in the Higgs potential [46]. These symmetries $\mathcal{P}_{I, I I}$ along with $Q_{6}$ enable us to reduce significantly the number of parameters in the fermion mass matrices. This reduction

${ }^{1}$ The finite group $Q_{6}$ was also considered by Frampton and Kong in [3]. See [7] for the tensor product.

The same model exists for $Q_{2 N}$ if $N$ is odd and a multiple of 3 . 
of parameters leads to a sum rule involving quark masses and mixing parameters [7]. We assume that CP violation is spontaneously broken, which is perhaps more satisfying than the usual assumption of explicit $\mathrm{CP}$ violation. Nevertheless, the dominant source of $\mathrm{CP}$ violation in the quark sector is the Kobayashi-Maskawa mechanism. With the spontaneous breaking of $\mathrm{CP}$, the $\mathrm{CP}$ problem that generically exists in the SSB sector can be softened in a rather simple way [7, 11, 41, 44, 46].

TABLE I: Particle content of the $Q_{6}$ model along with their transformation rule under $Q_{6} \times Z_{4}$.

\begin{tabular}{|c||c|c|c|c|c|c|c|c|c|c|c|c|c|c|c|}
\hline & $Q$ & $L$ & $Q_{3}$ & $L_{3}$ & $U^{c}, D^{c}, N^{c}, E^{c}$ & $U_{3}^{c}, D_{3}^{c}$ & $N_{3}^{c}$ & $E_{3}^{c}$ & $\Phi^{u, d}$ & $\Phi_{3}^{u, d}$ & $S$ & $S_{3}$ & $T$ & $T_{3}$ & $U$ \\
\hline$Q_{6}$ & 2 & $2^{\prime}$ & $1^{\prime}$ & 1 & $2^{\prime}$ & $1^{\prime \prime \prime}$ & $1^{\prime \prime}$ & 1 & $2^{\prime}$ & $1^{\prime \prime \prime}$ & 2 & 1 & $2^{\prime}$ & $1^{\prime}$ & 1 \\
\hline$Z_{4}$ & $-i$ & $-i$ & $-i$ & $-i$ & 1 & 1 & 1 & 1 & $i$ & $i$ & -1 & -1 & + & + & + \\
\hline$R$ & - & - & - & - & - & - & - & - & + & + & + & + & + & + & + \\
\hline
\end{tabular}

\section{A. The Higgs sector}

A certain set of SM singlet Higgs fields is needed to break $Q_{6}$ flavor symmetry spontaneously while avoiding pseudo NG bosons [46]. The minimal such set involves $\mathbf{2}, \mathbf{2}^{\prime}, \mathbf{1}^{\prime}$ and two 1's of $Q_{6}$. The SM singlet $S^{\prime}$ 's mix the $Q_{6}$ doublets $\Phi^{u, d}$ with the $Q_{6}$ singlets $\Phi_{3}^{u, d}$. An accidental $O(2)$ symmetry, which would exist without the $Q_{6}$ doublet $T$ in the Higgs potential, is violated by the cubic coupling of $T$. The $T_{3}$ is needed for the Majorana mass of $N_{3}^{c}$, and the $Q_{6}$ singlet $U$ is introduced to generate spontaneous CP violation and to induce spontaneous symmetry breaking of $Q_{6} \times Z_{4}$ within the SM singlet sector.

The most general Higgs superpotential involving the Higgs multiplets invariant under the $Q_{6} \times Z_{4}$ symmetry along with the matter parity has the form $W_{\text {Higgs }}=W_{U}+W_{S T}+W_{H}$, where

$$
\begin{aligned}
W_{U}= & \mu_{U} U^{2}+\lambda U^{3}+\left(\lambda_{1} S_{3}^{2}+\lambda_{2} T_{3}^{2}+\lambda_{3} T \cdot T\right) U, \\
W_{S T}= & \mu_{S_{3}} S_{3}^{2}+\mu_{T} T \cdot T+\mu_{T_{3}} T_{3}^{2}+\lambda_{3}^{\prime} T \cdot(T \otimes T) \\
& +\lambda_{1}^{\prime}\left[-2 S_{2} S_{1} T_{1}+\left(S_{1}^{2}-S_{2}^{2}\right) T_{2}\right]+\lambda_{2}^{\prime} S \cdot S T_{3}, \\
W_{H}= & \lambda_{1}^{\prime \prime} \Phi_{3}^{u}\left(\Phi^{d} * S\right)+\lambda_{2}^{\prime \prime}\left(\Phi^{u} * S\right) \Phi_{3}^{d}+\lambda_{3}^{\prime \prime}\left(\Phi^{u} \cdot \Phi^{d}\right) S_{3}
\end{aligned}
$$

with $x \cdot y=x_{1} y_{1}+x_{2} y_{2}, x * y=x_{1} y_{2}+x_{2} y_{1}, x \star y=x_{1} y_{2}-x_{2} y_{1}, x \cdot(y \otimes z)=$ $x_{1}\left(-y_{1} z_{1}+y_{2} z_{2}\right)+x_{2}\left(y_{1} z_{2}+y_{2} z_{1}\right)$. The Higgs potential contains $F$-terms derived from Eqs. (23) $-(25), D$-terms associated with $S U(2)_{L} \times U(1)_{Y}$ gauge symmetry, and the following SSB Lagrangian $^{2}$

$$
\begin{aligned}
\mathcal{L}_{\text {soft }} & =m_{U}^{2}|U|^{2}+m_{S}^{2}\left(\left|S_{1}\right|^{2}+\left|S_{2}\right|^{2}\right)+m_{S_{3}}^{2}\left|S_{3}\right|^{2}+m_{T}^{2}\left(\left|T_{1}\right|^{2}+\left|T_{2}\right|^{2}\right)+m_{T_{3}}^{2}\left|T_{3}\right|^{2} \\
& +m_{h_{3}^{u}}^{2}\left|h_{3}^{u}\right|^{2}+m_{h_{3}^{d}}^{2}\left|h_{3}^{d}\right|^{2}+m_{h^{u}}^{2}\left(\left|h_{1}^{u}\right|^{2}+\left|h_{2}^{u}\right|^{2}\right)+m_{h^{d}}^{2}\left(\left|h_{1}^{d}\right|^{2}+\left|h_{2}^{d}\right|^{2}\right) \\
& +\left\{B_{U} U^{2}+B_{S_{3}} S_{3}^{2}+B_{T} T \cdot T+B_{T_{3}} T_{3}^{2}\right.
\end{aligned}
$$

\footnotetext{
${ }^{2}$ We use the same symbol for the scalar components as the superfields for $T, S$ and $U$.
} 


$$
\begin{aligned}
& +\left[A U^{2}+A_{1} S_{3}^{2}+A_{2} T_{3}^{2}+A_{3}(T \cdot T)\right] U+A_{3}^{\prime} T \cdot(T \otimes T) \\
& +A_{1}^{\prime}\left[-2 S_{2} S_{1} T_{1}+\left(S_{1}^{2}-S_{2}^{2}\right) T_{2}\right]+A_{2}^{\prime} S \cdot S T_{3} \\
& \left.+A_{1}^{\prime \prime} h_{3}^{u}\left(h^{d} * S\right)+A_{2}^{\prime \prime}\left(h^{u} * S\right) h_{3}^{d}+A_{3}^{\prime \prime}\left(h^{u} \cdot h^{d}\right) S_{3}+\text { h.c. }\right\}
\end{aligned}
$$

We assume CP invariance, which implies that all the Yukawa couplings and the parameters in the Higgs potential are real. The Higgs potential derived from (23) -(25), and (26) including the $D$-terms admits two interesting minima which leave two separate discrete symmetries $\mathcal{P}_{I}$ and $\mathcal{P}_{I I}$ unbroken:

$$
\begin{aligned}
\mathcal{P}_{I}: & h_{1}^{u} \leftrightarrow h_{2}^{u}, h_{1}^{d} \leftrightarrow h_{2}^{d}, S_{1} \leftrightarrow S_{2}, T_{2} \rightarrow-T_{2}, \\
& h_{3}^{u} \rightarrow h_{3}^{u}, h_{3}^{d} \rightarrow h_{3}^{d}, S_{3} \rightarrow S_{3}, T_{1} \rightarrow T_{1}, T_{3} \rightarrow T_{3}, \quad U \rightarrow U, \\
\mathcal{P}_{I I}: & h_{1}^{u} \leftrightarrow h_{2}^{u *}, h_{1}^{d} \leftrightarrow h_{2}^{d *}, S_{1} \leftrightarrow S_{2}^{*}, T_{2} \rightarrow-T_{2}^{*}, \\
& h_{3}^{u} \rightarrow h_{3}^{u *}, h_{3}^{d} \rightarrow h_{3}^{d *}, S_{3} \rightarrow S_{3}^{*}, \quad T_{1} \rightarrow T_{1}^{*}, T_{3} \rightarrow T_{3}^{*}, U \rightarrow U^{*} .
\end{aligned}
$$

The VEVs of the various Higgs fields can be consistently chosen such that these symmetries remains unbroken. We have explicitly found local minima at which CP and $Q_{6} \times Z_{4}$ are spontaneously broken. We however will not present the full analysis of the potential. Furthermore, in the following discussions of the paper we consider only the second case $\mathcal{P}_{I I}$. The $\mathcal{P}_{I}$ invariant case works in a similar way.

The $\mathcal{P}_{I I}$ invariance enables us to choose a ground state given by

$$
\begin{aligned}
& \left\langle h_{1}^{u}\right\rangle=v_{1}^{u} e^{-i \phi^{u}},\left\langle h_{2}^{u}\right\rangle=v_{1}^{u} e^{i \phi^{u}},\left\langle h_{1}^{d}\right\rangle=v_{1}^{d} e^{-i \phi^{d}},\left\langle h_{2}^{d}\right\rangle=v_{1}^{d} e^{i \phi^{d}}, \\
& \left\langle h_{3}^{u}\right\rangle=v_{3}^{u},\left\langle h_{3}^{d}\right\rangle=v_{3}^{d},\left\langle S_{1}\right\rangle=v_{S} e^{-i \phi_{S}},\left\langle S_{2}\right\rangle=v_{S} e^{i \phi_{S}},\left\langle S_{3}\right\rangle=v_{S_{3}}, \\
& \left\langle T_{1}\right\rangle=v_{T_{1}},\left\langle T_{2}\right\rangle=-i v_{T_{2}},\left\langle T_{3}\right\rangle=v_{T_{3}},\langle U\rangle=v_{U},
\end{aligned}
$$

where the complex phases are all explicitly displayed. Note that there are only three phases, $\phi_{S}, \phi_{u}$ and $\phi_{d}$ in the VEVs, along with a purely imaginary VEV of $T_{2}$. To proceed we introduce:

$$
Y_{+}^{u, d}=\frac{1}{\sqrt{2}}\left(\Phi_{1}^{u, d}+\Phi_{2}^{u, d}\right), Y_{-}^{u, d}=\frac{i}{\sqrt{2}}\left(\Phi_{1}^{u, d}-\Phi_{2}^{u, d}\right),
$$

whose VEVs are given by $\left\langle Y_{+}^{u, d}\right\rangle=\sqrt{2} v_{1}^{u, d} \cos \phi^{u, d},\left\langle Y_{-}^{u, d}\right\rangle=\sqrt{2} v_{1}^{u, d} \sin \phi^{u, d}$. Then we redefine them as

$$
\left(\begin{array}{c}
\Phi_{L}^{u} \\
\Phi_{H}^{u} \\
\Phi_{-}^{u}
\end{array}\right)=\left(\begin{array}{ccc}
s_{\gamma^{u}} c_{\phi^{u}} & s_{\gamma^{u}} s_{\phi^{u}} & c_{\gamma^{u}} \\
c_{\gamma^{u}} c_{\phi^{u}} & c_{\gamma^{u}} s_{\phi^{u}} & -s_{\gamma^{u}} \\
-s_{\phi^{u}} & c_{\phi^{u}} & 0
\end{array}\right)\left(\begin{array}{c}
Y_{+}^{u} \\
Y_{-}^{u} \\
\Phi_{3}^{u}
\end{array}\right),
$$

where

$$
\begin{aligned}
& c_{\gamma^{u}}=\cos \gamma^{u}=\sqrt{2} v_{3}^{u} / v_{u}, s_{\gamma^{u}}=\sin \gamma^{u}=2 v_{1}^{u} / v_{u}, \\
& c_{\phi^{u}}=\sin \phi^{u}, s_{\phi^{u}}=\sin \phi^{u}, v_{u}=\sqrt{2\left(v_{3}^{u}\right)^{2}+4\left(v_{1}^{u}\right)^{2}},
\end{aligned}
$$

and similarly for the down sector. As we see from Eqs. (30)- (32), only $\Phi_{L}^{u}$ and $\Phi_{L}^{d}$ have a nonvanishing VEV. According to (10), the new Higgs doublet supermultiplets are defined as

$$
\Phi_{I}^{u}=\left(h_{I}^{u}, \tilde{h}_{I}^{u}\right)=\left(\begin{array}{cc}
h_{I}^{u+} & \tilde{h}_{I}^{u+} \\
h_{I}^{u 0} & \tilde{h}_{I}^{u 0}
\end{array}\right), \Phi_{I}^{d}=\left(h_{I}^{d}, \tilde{h}_{I}^{d}\right)=\left(\begin{array}{cc}
h_{I}^{d 0} & \tilde{h}_{I}^{d 0} \\
h_{I}^{d-} & \tilde{h}_{I}^{d-}
\end{array}\right)
$$


with $I=L, H,-$. As we see from Eq. (32), $h_{L}^{u 0}$ and $h_{L}^{d 0}$ are assumed to have a nonvanishing VEV : $\left\langle h_{L}^{u 0, d 0}\right\rangle=v_{u, d} / \sqrt{2}$. The light and heavy MSSM-like Higgs scalars are then given by

$$
\begin{aligned}
\frac{1}{\sqrt{2}}(H+i A) & =\left(h_{L}^{d 0}\right)^{*} s_{\beta}-\left(h_{L}^{u 0}\right) c_{\beta}, \frac{1}{\sqrt{2}}(v+h+i G)=\left(h_{L}^{d 0}\right)^{*} c_{\beta}+\left(h_{L}^{u 0}\right) s_{\beta}, \\
G^{+} & =-\left(h_{L}^{d-}\right)^{*} c_{\beta}+\left(h_{L}^{u+}\right) s_{\beta}, H^{+}=\left(h_{L}^{d-}\right)^{*} s_{\beta}+h_{L}^{u} c_{\beta}, \\
v & =\sqrt{v_{u}^{2}+v_{d}^{2}}(\simeq 246 \mathrm{GeV}), \tan \beta=v_{u} / v_{d},
\end{aligned}
$$

where $c_{\beta}=\cos \beta, s_{\beta}=\sin \beta, G$ and $G^{+}$are the NG fields, and we assumed that the mass of $H$ and $A$ is much larger than $M_{Z}$. As in the case of the MSSM, the couplings of $\Phi_{L}^{u, d}$ are flavor-diagonal, while the extra heavy neutral fields $h_{-}^{u 0, d 0}=\left(\varphi_{-}^{u, d}+i \chi_{-}^{u, d}\right) / \sqrt{2}, h_{H}^{u 0, d 0}=$ $\left(\varphi_{H}^{u, d}+i \chi_{H}^{u, d}\right) / \sqrt{2}$ can have flavor-changing couplings.

The SM like Higgs boson $h$ defined in (35) is the lightest Higgs boson in this model. The tree-level upper bound of its mass has due to the cubic superpotential (25) an extra contribution in addition to the MSSM one:

$$
\begin{aligned}
m_{h}^{2} \lesssim & M_{Z}^{2} c_{2 \beta}^{2}+\frac{1}{2} v^{2} s_{2 \beta}^{2}\left[c_{\gamma^{u}}^{2} s_{\gamma^{d}}^{2}\left(\lambda_{1}^{\prime \prime}\right)^{2}+\frac{1}{2} c_{2 \gamma^{u}} s_{2 \gamma^{d}} \cos \left(\phi^{u}-\phi^{d}\right) \lambda_{1}^{\prime \prime} \lambda_{2}^{\prime \prime}\right. \\
& \left.+s_{\gamma^{u}}^{2}\left[c_{\gamma^{d}}^{2}\left(\lambda_{2}^{\prime \prime}\right)^{2}+s_{\gamma^{d}}^{2} \cos \left(\phi^{u}+\phi^{d}\right)\left(\lambda_{3}^{\prime \prime}\right)^{2}\right]\right],
\end{aligned}
$$

where $c_{\gamma^{u}}$, etc. are defined in (32). So with the radiative correction included [50] one can bring $m_{h}$ easily to $\sim 125 \mathrm{GeV}$, around which an excess of events is reported at LHC [51, 52].

By integrating out the SM singlet supermultiplets, we can obtain the effective theory. The Higgs superpotential of the effective theory can be written as

$$
W_{I I}^{\mathrm{eff}}=\mu_{11}\left(\Phi_{1}^{u} \Phi_{1}^{d}+\Phi_{2}^{u} \Phi_{2}^{d}\right)+\mu_{32} \Phi_{3}^{u} \Phi_{2}^{d}+\mu_{32}^{*} \Phi_{3}^{u} \Phi_{1}^{d}+\mu_{13} \Phi_{1}^{u} \Phi_{3}^{d}+\mu_{13}^{*} \Phi_{2}^{u} \Phi_{3}^{d},
$$

where $\mu_{11}=\mu_{22}$ is real. $W_{I I}^{\text {eff }}$ yields a $\mathcal{P}_{I I}$ invariant scalar potential, but breaks $Q_{6}$ flavor symmetry softly. The soft breaking is consistent with $Q_{6}$ according to (Th1). In terms of the redefined Higgs superfields (31), $W_{I I}^{\text {eff }}$ becomes

$$
\begin{aligned}
W_{I I}^{\mathrm{eff}} & =\mu_{L} \Phi_{L}^{u} \Phi_{L}^{d}+\mu_{L H} \Phi_{L}^{u} \Phi_{H}^{d}+\mu_{H L} \Phi_{H}^{u} \Phi_{L}^{d}+\mu_{H} \Phi_{H}^{u} \Phi_{H}^{d}+\mu_{-} \Phi_{-}^{u} \Phi_{-}^{d} \\
& +\mu_{-L} \Phi_{-}^{u} \Phi_{L}^{d}+\mu_{L-} \Phi_{L}^{u} \Phi_{-}^{d}+\mu_{-H} \Phi_{-}^{u} \Phi_{H}^{d}+\mu_{H-} \Phi_{H}^{u} \Phi_{-}^{d}
\end{aligned}
$$

and similarly the SSB Lagrangian can be written as

$$
\begin{aligned}
\mathcal{L}_{I I \text { soft }}^{\mathrm{eff}} & =m_{u L}^{2}\left|h_{L}^{u}\right|^{2}+\left[m_{u L H}^{2} h_{L}^{u *} h_{H}^{u}+h . c .\right]+m_{u H}^{2}\left|h_{H}^{u}\right|^{2}+m_{u-}^{2}\left|h_{-}^{u}\right|^{2} \\
& +m_{d L}^{2}\left|h_{L}^{d}\right|^{2}+\left[m_{d L H}^{2} h_{L}^{d *} h_{H}^{d}+h . c .\right]+m_{d H}^{2}\left|h_{H}^{u}\right|^{2}+m_{d-}^{2}\left|h_{-}^{d}\right|^{2} \\
& +\left[B_{L} h_{L}^{u} h_{L}^{d}+B_{L H} h_{L}^{u} h_{H}^{d}+B_{H L} h_{H}^{u} h_{L}^{d}+B_{H} h_{H}^{u} h_{H}^{d}+B_{-} h_{-}^{u} h_{-}^{d}+h . c .\right] \\
& +\left[B_{-L} h_{-}^{u} h_{L}^{d}+B_{L-} h_{L}^{u} h_{-}^{d}+B_{-H} h_{-}^{u} h_{H}^{d}+B_{H-} h_{H}^{u} h_{-}^{d} \text { h.c. }\right] .
\end{aligned}
$$

Note that the parameters appearing in Eqs. (40) and (41) are all real. This is because all the fields can be redefined without a non-trivial phase rotation in such way that their VEVs become real. That is, CP is restored in the Higgs sector. Because of the restored $\mathrm{CP}$ invariance in the Higgs sector, the CP even and odd fields do not mix at the tree level. 
Accordingly, the mass matrix for the neutral CP even Higgs bosons assumes the form

$$
\mathbf{M}_{N \text { Heven }}^{2}=\left(\begin{array}{cccccc}
c_{2 \beta}^{2} M_{z}^{2} & -c_{2 \beta} s_{2 \beta} M_{z}^{2} & 0 & 0 & 0 & 0 \\
-c_{2 \beta} s_{2 \beta} M_{z}^{2} & \frac{2 B_{L}}{S_{2 \beta}}+s_{2 \beta}^{2} M_{z}^{2} & -\frac{\hat{m}_{u L H}^{2}}{c_{\beta}} & \frac{\hat{m}_{d L H}^{2}}{s_{\beta}} & \frac{\mu_{1}^{2}}{c_{\beta}} & -\frac{\mu_{2}^{2}}{s_{\beta}} \\
0 & -\frac{\hat{m}_{u L H}^{2}}{c_{\beta}} & -\hat{m}_{u H}^{2} & -B_{H} & \mu_{3}^{2} & -B_{H-} \\
0 & \frac{\hat{m}_{d L H}^{2}}{s_{\beta}} & -B_{H} & -\hat{m}_{d H}^{2} & -B_{-H} & \mu_{4}^{2} \\
0 & \frac{\mu_{1}^{2}}{c_{\beta}} & \mu_{3}^{2} & -B_{-H} & -\hat{m}_{u-}^{2} & -B_{-} \\
0 & -\frac{\mu_{2}^{2}}{s_{\beta}} & -B_{H-} & \mu_{4}^{2} & -B_{-} & -\hat{m}_{d-}^{2}
\end{array}\right)
$$

in the basis $\left(h, H, \varphi_{H}^{u}, \varphi_{H}^{d}, \varphi_{-}^{u}, \varphi_{-}^{d}\right)$, where

$$
\begin{aligned}
\mu_{1}^{2} & =\mu_{L-} \mu_{-}+\mu_{L H} \mu_{-H}+\mu_{L} \mu_{-L}, \mu_{2}^{2}=\mu_{H L} \mu_{H-}+\mu_{L} \mu_{L-}+\mu_{-} \mu_{-L}, \\
-\hat{m}_{u L H}^{2} & =-m_{u L H}^{2}+\mu_{H L} \mu_{L}+\mu_{H} \mu_{L H}+\mu_{H-} \mu_{L-}, \\
-\hat{m}_{d L H}^{2} & =-m_{d L H}^{2}+\mu_{H} \mu_{H L}+\mu_{L} \mu_{L-}+\mu_{-H} \mu_{-L}, \\
\mu_{3}^{2} & =\mu_{H-} \mu_{-}+\mu_{H} \mu_{-H}+\mu_{H L} \mu_{-L}, \mu_{4}^{2}=\mu_{H} \mu_{H-}+\mu_{L H} \mu_{L-}+\mu_{-} \mu_{-H}, \\
-\hat{m}_{u(d) H}^{2} & =-m_{u(d) H}^{2}+\mu_{H}^{2}+\mu_{H L(L H)}^{2}+\mu_{H-(-H)}^{2}-(+) \frac{1}{2} c_{2 \beta} M_{z}^{2}, \\
-\hat{m}_{u(d)-}^{2} & =-m_{u(d)-}^{2}+\mu_{-}^{2}+\mu_{-H(H-)}^{2}+\mu_{-L(L-)}^{2}-(+) \frac{1}{2} c_{2 \beta} M_{z}^{2} .
\end{aligned}
$$

The mass matrix for the $\mathrm{CP}$ odd neutral Higgs bosons is found to be

$$
\mathbf{M}_{N H o d d}^{2}=\left(\begin{array}{ccccc}
\frac{B_{L}}{c_{\beta} s_{\beta}} & -\frac{\hat{m}_{u L H}^{2}}{c_{\beta}} & \frac{\hat{m}_{d L H}^{2}}{s_{\beta}} & \frac{\mu_{1}^{2}}{c_{\beta}} & \frac{\mu_{2}^{2}}{s_{\beta}} \\
-\frac{\hat{m}_{u L H}^{2}}{c_{\beta}}-\hat{m}_{u H}^{2}+c_{\theta_{W}}^{2} c_{2 \beta} M_{Z}^{2} & B_{H} & \mu_{3}^{2} & B_{H-} \\
\frac{\hat{m}_{d L H}^{2}}{s_{\beta}} & B_{H} & -\hat{m}_{d H}^{2}-c_{\theta_{W}}^{2} c_{2 \beta} M_{Z}^{2} & B_{-H} & \mu_{4}^{2} \\
\frac{\mu_{1}^{2}}{c_{\beta}} & \mu_{3}^{2} & B_{-H} & -\hat{m}_{u-}^{2}+c_{\theta_{W}}^{2} c_{2 \beta} M_{Z}^{2} & B_{-} \\
\frac{\mu_{2}^{2}}{s_{\beta}} & B_{H-} & \mu_{4}^{2} & B_{-} & -\hat{m}_{d-}^{2}-c_{\theta_{W}}^{2} c_{2 \beta} M_{Z}^{2}
\end{array}\right)
$$

in the basis $\left(A, \chi_{H}^{u 0}, \chi_{H}^{d 0}, \chi_{-}^{u 0}, \chi_{-}^{d 0}\right)$. Similarly, we obtain the mass matrix of the charged Higgs bosons:

$$
\mathbf{M}_{C}^{2}=\left(\begin{array}{ccccc}
\frac{B_{L}}{c_{\beta} s_{\beta}} & -\frac{\hat{m}_{u L H}^{2}}{c_{\beta}} & -\frac{\hat{m}_{d L H}^{2}}{s_{\beta}} & \frac{\mu_{1}^{2}}{c_{\beta}} & \frac{\mu_{2}^{2}}{s_{\beta}} \\
-\frac{\hat{m}_{u L H}^{2}}{c_{\beta}} & -\hat{m}_{u H}^{2} & B_{H} & \mu_{3}^{2} & B_{H-} \\
-\frac{\hat{m}_{d L H}^{2}}{s_{\beta}} & B_{H} & -\hat{m}_{d H}^{2} & B_{-H} & \mu_{4}^{2} \\
\frac{\mu_{1}^{2}}{c_{\beta}} & \mu_{3}^{2} & B_{-H} & -\hat{m}_{u-}^{2} & B_{-} \\
\frac{\mu_{2}^{2}}{s_{\beta}} & B_{H-} & \mu_{4}^{2} & B_{-} & -\hat{m}_{d-}^{2}
\end{array}\right)
$$

in the basis $\left(H^{+}, h_{H}^{u+}, h_{H}^{d-*}, h_{-}^{u+}, h_{-}^{d-*}\right)$.

\section{B. The Yukawa sector}

The most general, renormalizable, $Q_{6} \times Z_{4} \times R$ invariant superpotential $W_{Y}$ in the Yukawa sector is given by ${ }^{3} W_{Y}=W_{Q}+W_{L}$, where $W_{Q}$ and $W_{L}$ are given in (7) with the Yukawa

\footnotetext{
${ }^{3}$ The $Q_{6} \times Z_{4} \times R$ assignment is given in Table 1 .
} 
matrices

$$
\begin{gathered}
\mathbf{Y}^{u 1(d 1)=}\left(\begin{array}{ccc}
0 & 0 & 0 \\
0 & 0 & Y_{b}^{u(d)} \\
0 & Y_{b^{\prime}}^{u(d)} & 0
\end{array}\right), \mathbf{Y}^{u 2(d 2)}=\left(\begin{array}{ccc}
0 & 0 & Y_{b}^{u(d)} \\
0 & 0 & 0 \\
-Y_{b^{\prime}}^{u(d)} & 0 & 0
\end{array}\right), \mathbf{Y}^{u 3(d 3)}=\left(\begin{array}{ccc}
0 & Y_{c}^{u(d)} & 0 \\
Y_{c}^{u(d)} & 0 & 0 \\
0 & 0 & Y_{a}^{u(d)}
\end{array}\right) \\
\mathbf{Y}^{e 1}=\left(\begin{array}{ccc}
-Y_{c}^{e} & 0 & Y_{b}^{e} \\
0 & Y_{c}^{e} & 0 \\
Y_{b^{\prime}}^{e} & 0 & 0
\end{array}\right), \mathbf{Y}^{e 2}=\left(\begin{array}{ccc}
0 & Y_{c}^{e} & 0 \\
Y_{c}^{e} & 0 & Y_{b}^{e} \\
0 & Y_{b^{\prime}}^{e} & 0
\end{array}\right), \mathbf{Y}^{e 3}=0 \\
\mathbf{Y}^{\nu 1}=\left(\begin{array}{ccc}
-Y_{c}^{\nu} & 0 & 0 \\
0 & Y_{c}^{\nu} & 0 \\
Y_{b^{\prime}}^{\nu} & 0 & 0
\end{array}\right), \mathbf{Y}^{\nu 2}=\left(\begin{array}{ccc}
0 & Y_{c}^{\nu} & 0 \\
Y_{c}^{\nu} & 0 \\
0 & Y_{b^{\prime}}^{\nu} & 0
\end{array}\right), \mathbf{Y}^{\nu 3}=\left(\begin{array}{ccc}
0 & 0 & 0 \\
0 & 0 & 0 \\
0 & 0 & Y_{a}^{\nu}
\end{array}\right)
\end{gathered}
$$

All the parameters appearing above are real, because we assume that CP is spontaneously broken. From the Yukawa interactions along with the Yukawa matrices (49)-(51) and the VEV structure of the $\mathcal{P}_{I I}$ invariant case (given in (29), we obtain the fermion mass matrices. As we will see that the resulting mass matrix of the quarks is of a nearest neighbor interaction (NNI) type which is one of the successful Ansätze for the quark mass matrix [53, 54] ${ }^{4}$. The $Q_{6}$ assignment of the fermions is so chosen that the NNI type of the mass matrix occurs from flavor symmetry ( see also [56] ). The Majorana mass term for $N^{c}$ is given by $W_{M}=\frac{1}{2} M_{1}\left(N_{1}^{c} N_{1}^{c}+N_{2}^{c} N_{2}^{c}\right)+\lambda_{M}<T_{3}>N_{3}^{c} N_{3}^{c}$. We first discuss the quark sector and then the lepton sector in the following sections.

\section{FERMION MASS AND MIXING}

\section{A. The quark sector and the Cabibbo-Kobayashi-Maskawa (CKM) mixing}

In the background $\mathcal{P}_{I I}$, the fermion mass matrices $\mathbf{m}_{u, d}$ take the form

$$
\mathbf{m}_{u, d}=\left(\begin{array}{ccc}
0 & C_{u, d} & \frac{B_{u, d}}{\sqrt{2}} e^{-i \phi^{u, d}} \\
C_{u, d} & 0 & \frac{B_{u, d}}{\sqrt{2}} e^{i \phi^{u, d}} \\
-\frac{B_{u, d}^{\prime}}{\sqrt{2}} e^{-i \phi^{u, d}} & \frac{B_{u, d}^{\prime}}{\sqrt{2}} e^{i \phi^{u, d}} & A_{u, d}
\end{array}\right),
$$

where $A_{u, d}=Y_{a}^{u, d} v_{3}^{u, d}, B_{u, d}=\sqrt{2} Y_{b}^{u, d} v_{1}^{u, d}, B_{u, d}^{\prime}=\sqrt{2} Y_{b^{\prime}}^{u, d} v_{1}^{u, d}, C_{u, d}=Y_{c}^{u, d} v_{3}^{u, d}$. The phases in the matrix of Eq. (52) can be factorized as $\mathbf{m}_{u, d}^{r}=P_{u, d} \mathbf{m}_{u, d} P_{u, d}$, where

$$
P_{u, d}=\operatorname{diag} .\left\{e^{i \phi^{u, d}}, e^{-i \phi^{u, d}}, 1\right\}
$$

Then we do a 45 degrees rotation in the (1-2) plane to bring $\mathbf{m}_{u, d}^{r}$ into

$$
\hat{\mathbf{m}}_{u, d}=R_{L}^{T} \mathbf{m}_{u, d}^{r} R_{R}=\left(\begin{array}{ccc}
0 & C_{u, d} & 0 \\
-C_{u, d} & 0 & B_{u, d} \\
0 & B_{u, d}^{\prime} & A_{u, d}
\end{array}\right),
$$

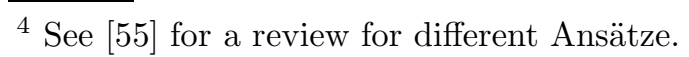


generating a non-trivial quark mixing matrix given by

$$
K=R_{L}^{T} P_{u}^{\dagger} P_{d} R_{L}=\left(\begin{array}{ccc}
\cos \phi & i \sin \phi & 0 \\
i \sin \phi & \cos \phi & 0 \\
0 & 0 & 1
\end{array}\right), \phi=\phi^{u}-\phi^{d}
$$

where

$$
R_{L}=\frac{1}{\sqrt{2}}\left(\begin{array}{ccc}
1 & 1 & 0 \\
-1 & 1 & 0 \\
0 & 0 & \sqrt{2}
\end{array}\right), R_{R}=\frac{1}{\sqrt{2}}\left(\begin{array}{ccc}
-1 & -1 & 0 \\
-1 & 1 & 0 \\
0 & 0 & \sqrt{2}
\end{array}\right)
$$

The CKM mixing matrix is then obtained as $V_{\mathrm{CKM}}=O_{u}^{T} K O_{d}$, where $O_{u, d}$ diagonalize the matrices of Eq. (54). An excellent fit is obtained with the following choice of parameters at $\mu=1 \mathrm{TeV}$ :

$$
\begin{aligned}
A_{u} / m_{t} & =7.031 \times 10^{-3}, B_{u} / m_{t}=-3.124 \times 10^{-3}, B_{u}^{\prime} / m_{t}=1.000, C_{u} / m_{t}=1.750 \times 10^{-3}, \\
A_{d} / m_{b} & =0.9200, B_{d} / m_{b}=0.04406, B_{d}^{\prime} / m_{b}=0.3899, C_{d} / m_{b}=4.203 \times 10^{-3}, \\
\phi & =0.08375 .
\end{aligned}
$$

The resulting quark masses at $\mu=1 \mathrm{TeV}$ are:

$$
m_{u}=0.910 \mathrm{MeV}, m_{c}=542 \mathrm{MeV}, m_{d}=2.21 \mathrm{MeV}, m_{s}=44.5 \mathrm{MeV} \text {, }
$$

where we have used $m_{t}=151.3 \mathrm{GeV}$ and $m_{b}=2.46 \mathrm{GeV}$. These values are to be compared with quark masses extrapolated from low energy scale to $\mu=1 \mathrm{TeV}$ [57]:

$$
\begin{aligned}
& m_{u}=0.85 \sim 1.55 \mathrm{MeV}, m_{d}=2.05 \sim 2.85 \mathrm{MeV}, \\
& m_{s}=39.6 \sim 64.4 \mathrm{MeV}, m_{c}=502 \sim 570 \mathrm{MeV}, \\
& m_{b}=2.39 \sim 2.53 \mathrm{GeV}, m_{t}=150.3 \sim 151.8 \mathrm{GeV},
\end{aligned}
$$

where we have updated the result of [57] by using the updated quark masses given in PDG 2011 [58], while neglecting the uncertainties due to the RG running. The input values of Eq. (57) give the output for the CKM parameters:

$$
\begin{aligned}
\lambda & =0.2260, A=0.792, \bar{\rho}=0.148, \bar{\eta}=0.331, \\
\sin 2 \beta & =0.675, \alpha=92.8[\mathrm{deg}], \beta=21.2[\mathrm{deg}], \gamma=65.9[\mathrm{deg}],
\end{aligned}
$$

which should be compared with the fit result of the CKMfitter group [59, 60]

$$
\begin{aligned}
\lambda & =0.22539_{-0.00095}^{+0.00062}, A=0.801_{-0.014}^{+0.026} \\
\bar{\rho} & =0.144_{-0.026}^{+0.023}, \bar{\eta}=0.343_{-0.014}^{+0.015}, \quad \sin 2 \beta=0.691_{-0.020}^{+0.020}, \\
\alpha & =90.9_{-4.1}^{+3.5}[\mathrm{deg}], \beta=21.84_{-0.76}^{+0.80}[\mathrm{deg}], \gamma=67.3_{-3.5}^{+4.2}[\mathrm{deg}]
\end{aligned}
$$

Fig. 1 shows the prediction in the $\bar{\rho}-\bar{\eta}$ plane (a) and in the $\beta-\gamma$ plane (b) for $\mathcal{P}_{I I}$. The CKMfitter group best-fit value (162) is indicated in these plots. We see from Eqs. (58) and Fig. 1 that the model $\mathcal{P}_{I I}$ reproduces the quark masses, CKM mixings and the CP violating phase in an excellent way. 


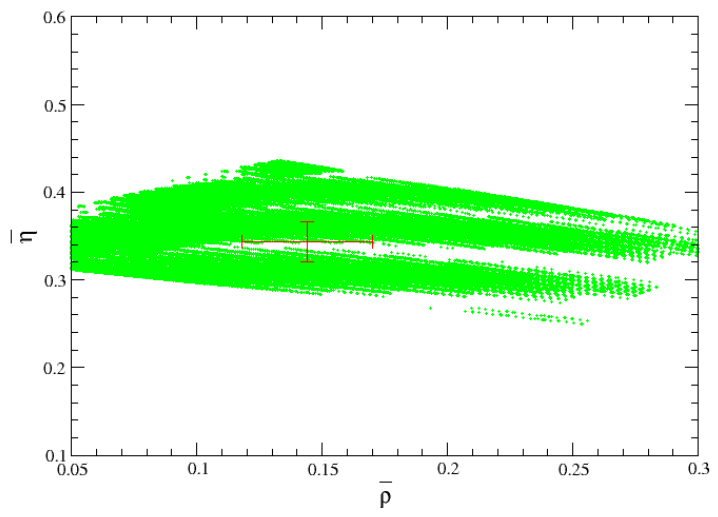

a)

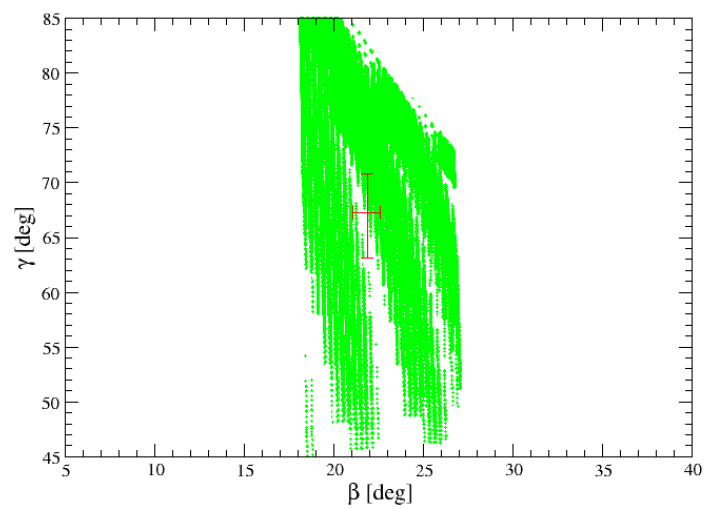

b)

FIG. 1: The predictions in the $\bar{\rho}-\bar{\eta}$ plane (a) and $\beta-\gamma$ plane (b) for the $\mathcal{P}_{I I}$ invariant model, where we have used as the input parameters; the quark masses, $\lambda$ and $A$ given in Eqs. (59) and (61), respectively. We also have imposed the constraints on the quark masses [58]: $2 m_{s} /\left(m_{u}+m_{d}\right)=22 \sim 30, m_{s} / m_{d}=17 \sim$ $22, m_{u} / m_{d}=0.35 \sim 0.60,(1 / 2)\left(m_{u}+m_{d}\right)(2 \mathrm{GeV})=(3.0 \sim 4.8) \mathrm{MeV}$. The crosses denote one $\sigma$ value given in [59].

\section{B. Lepton sector}

From the VEV structure of the $\mathcal{P}_{I I}$ invariant case, given in (29), we see that the Majorana mass term for $N_{3}^{c}$ is real, because $<T_{3}>$ is real: $M_{N}=\left(M_{1}, M_{1}, M_{3}=a_{\nu^{c}} v_{T_{3}}\right)$. The Dirac neutrino and charged lepton mass matrices are:

$$
\mathbf{m}_{\nu^{D}}=\left(\begin{array}{ccc}
-C_{\nu} e^{-i \phi^{u}} & C_{\nu} e^{i \phi^{u}} & 0 \\
C_{\nu} e^{i \phi^{u}} & C_{\nu} e^{-i \phi^{u}} & 0 \\
B_{\nu}^{\prime} e^{-i \phi^{u}} & B_{\nu}^{\prime} e^{i \phi^{u}} & A_{\nu}
\end{array}\right), \mathbf{m}_{\ell}=\left(\begin{array}{ccc}
-C_{\ell} e^{i \phi^{d}} & C_{\ell} e^{-i \phi^{d}} & B_{\ell} e^{i \phi^{d}} \\
C_{\ell} e^{-i \phi^{d}} & C_{\ell} e^{i \phi^{d}} & B_{\ell} e^{-i \phi^{d}} \\
B_{\ell}^{\prime} e^{i \phi^{d}} & B_{\ell}^{\prime} e^{-i \phi^{d}} & 0
\end{array}\right),
$$

which (by the seesaw formula) lead to the light neutrino Majorana mass matrix

$$
M_{\nu}^{\prime}=m_{0}\left(\begin{array}{ccc}
2 \rho_{2}^{2} \cos \left(2 \phi^{u}\right) & 0 & 2 i \rho_{2} \rho_{4} \sin \left(2 \phi^{u}\right) \\
0 & 2 \rho_{2}^{2} \cos \left(2 \phi^{u}\right) & 2 \rho_{2} \rho_{4} \\
2 i \rho_{2} \rho_{4} \sin \left(2 \phi^{u}\right) & 2 \rho_{2} \rho_{4} & -\rho_{3}^{2}+2 \rho_{4}^{2} \cos \left(2 \phi^{u}\right)
\end{array}\right),
$$



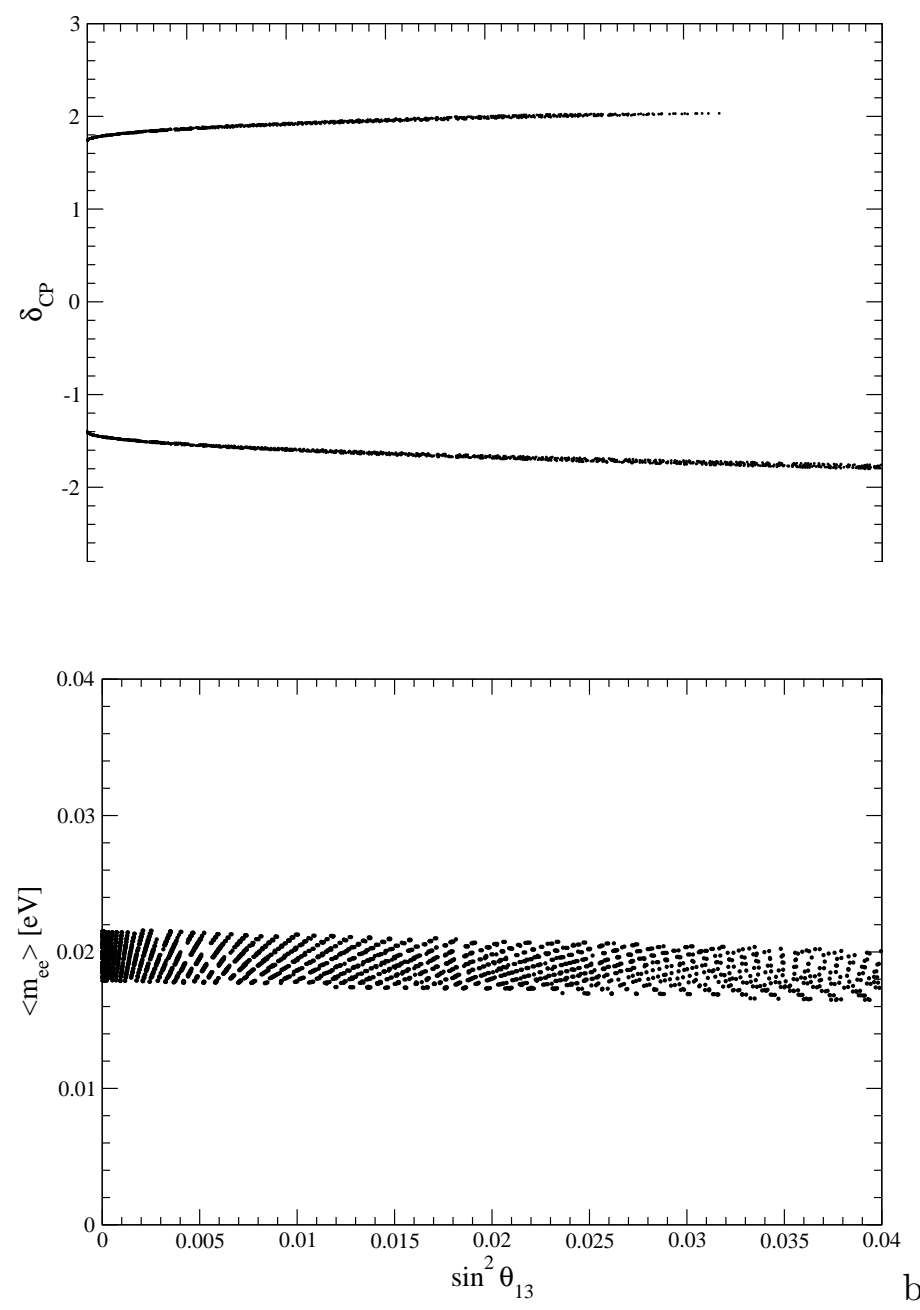

FIG. 2: The prediction in the $\sin ^{2} \theta_{13}-\delta_{C P}$ plane (a) and in the $\sin ^{2} \theta_{13}-<m_{e e}>$ plane (b) for $\mathcal{P}_{I I}$. We have used $\sin ^{2} \theta_{12}, \Delta m_{21}^{2}$ and $\Delta m_{23}^{2}$ given in (65) as the input values of the parameters, and $\phi_{d}=\phi_{u}-0.08375$.

where $\rho_{2}^{2}=\left(C_{\nu}\right)^{2} / M_{1}, \rho_{3}^{2}=-\left(A_{\nu}\right)^{2} / M_{3}, \rho_{4}^{2}=\left(B_{\nu}^{\prime}\right)^{2} / M_{1}$. We have assumed that $M_{1}$ is positive, while $M_{3}$ is negative. We make the matrix (64) real by redefining $\nu_{1}=i \nu_{1}^{\prime}$. The resulting mass matrix can be diagonalized by an orthogonal matrix. As for the charged lepton mass matrix $\mathbf{m}_{\ell}$, we can obtain hierarchical masses, e.g., $m_{e} \sim B_{\ell}^{\prime}, m_{\mu} \sim C_{\ell}, m_{\tau} \sim B_{\ell}$. Since the relative phase $\phi=\phi^{u}-\phi^{d}$ is fixed in the quark sector, there are seven independent parameters in the lepton sector. When $\phi^{u}=0$, the neutrino mass matrix above yields only a tiny $\sin ^{2} \theta_{13} \simeq m_{e} / m_{\mu} \sim 10^{-3}$ [5], which is too small [61 65]. For non-zero $\phi^{u}$, we obtain $\sin \theta_{13} \propto \sin 2 \phi^{u}$, which can be small or large. We use the three charged lepton masses, $\sin ^{2} \theta_{12}, \Delta m_{21}^{2}$ and $\Delta m_{23}^{2}$, and vary the value of $\phi^{u}$ while $\phi^{d}$ is set equal to $\phi^{u}-0.08375$ (see (57)), where the up-dated neutrino oscillation parameters [61] in which the recent results of T2K [62] and MINOS [63] experiments are included are:

$$
\begin{aligned}
\Delta m_{21}^{2} & =7.59_{-0.18}^{+0.20} \times 10^{-5} \mathrm{eV}^{2}, \Delta m_{23}^{2}=2.4_{-0.09}^{+0.08} \times 10^{-3} \mathrm{eV}^{2}, \\
\sin ^{2} \theta_{12} & =0.312_{-0.015}^{+0.017}, \sin ^{2} \theta_{13}=0.016_{-0.006}^{+0.008}, \sin ^{2} \theta_{23}=0.52 \pm 0.06 .
\end{aligned}
$$




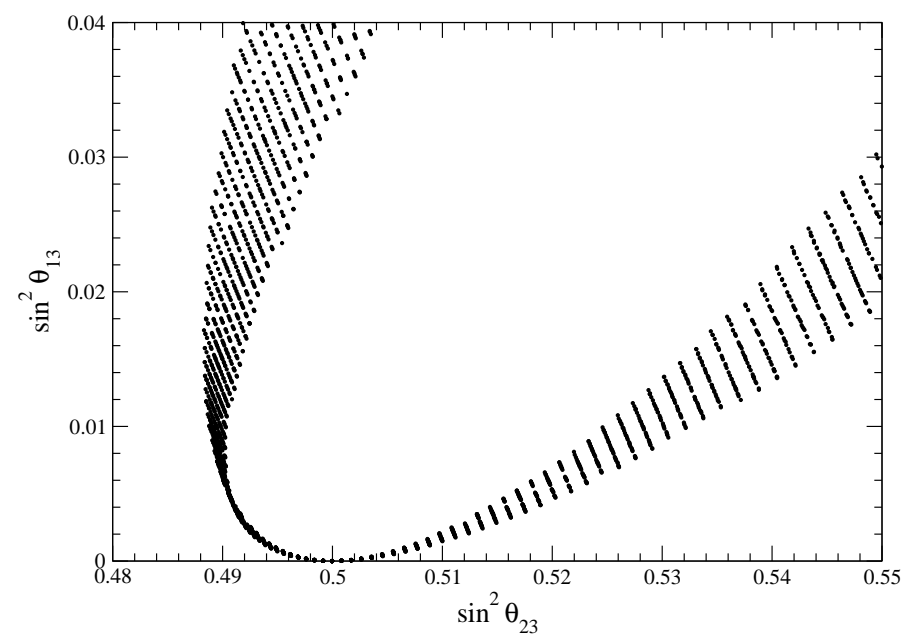

FIG. 3: $\sin ^{2} \theta_{13}$ against $\sin ^{2} \theta_{23}$ in the case of $\mathcal{P}_{I I}$ for the same input parameters as Fig. 2.

We below display the predictions of the model in different two dimensional planes. Fig. 2 (a) shows the Dirac phase $\delta_{C P}$ (in the convention of Ref. [58]) against $\sin ^{2} \theta_{13}$. We see that the model predicts nearly maximal $\mathrm{CP}$ violation. It is also possible to predict the effective neutrino mass $\left\langle m_{e e}>\right.$ as a function of $\sin ^{2} \theta_{13}$. If the mixing is tri-bimaximal [66], for instance, then $m_{\nu_{2}} \simeq \sqrt{\Delta m_{23}^{2}} 3 \sqrt{3} / 2 \sqrt{2} \simeq 0.09 \mathrm{eV}$. In Fig. 2 (b) we plot the prediction in the $\sin ^{2} \theta_{13}-<m_{e e}>$ plane. The deviation from the maximal mixing has terms proportional to $m_{e} / m_{\mu}$ and to $\sin 2 \phi_{u}$. In Fig. 3 we plot $\sin ^{2} \theta_{13}$ against $\sin ^{2} \theta_{23}$. Once the $\sin ^{2} \theta_{13}$ and $\sin ^{2} \theta_{23}$ are measured, the model prediction can be tested by experiments (see [67] and [68]).

\section{SUPPRESSING FCNCS BY FLAVOR SYMMETRY AND CP VIOLATIONS BY SELF-ALIGNMENT OF PHASES}

\section{A. Tree-level FCNCs and the Cheng-Sher mechanism}

Before we go to FCNCs coming from the supersymmetry breaking, we shall demonstrate that the Cheng-Sher mechanism [23] to suppress the tree-level FCNCs is partially embedded in the $Q_{6}$ model (see also [56]). Consider the mass difference of the neutral Kaons $\Delta M_{K} \simeq$ $3.5 \times 10^{-15} \mathrm{GeV}$, for instance, and assume that there is a neutral Higgs boson $H$ with mass $M_{H}$ which has a flavor changing Yukawa coupling with the down and strange quarks: $\left(Y_{d s} \bar{d}_{L} s_{R}+Y_{s d} \bar{s}_{L} d_{R}\right) H+$ h.c.. Then the contribution to $\Delta M_{K}$ can be estimated as [34]

$$
\Delta M_{K}=2\left|<\bar{K}^{0}\right| \bar{s}_{R} d_{L} \bar{s}_{L} d_{R}\left|K^{0}>\right|\left(\frac{\left|Y_{d s} Y_{s d}^{*}\right|}{M_{H}^{2}}\right)=\frac{1}{2} f_{K}^{2} B_{K}^{\prime} M_{K}\left(\frac{M_{K}}{m_{s}+m_{d}}\right)^{2}\left(\frac{Y_{d s} Y_{s d}^{*}}{M_{H}^{2}}\right),
$$

where $f_{K} \simeq 0.16 \mathrm{GeV}$ is the Kaon decay constant, $B_{K}^{\prime}$ is a bag parameter for the matrix element in (66). Using $M_{K} \simeq 0.5 \mathrm{GeV}, m_{s} \simeq 0.1 \mathrm{GeV}$ and $m_{d} \simeq 5 \times 10^{-3} \mathrm{GeV}$, we obtain

$$
\Delta M_{K} \simeq 0.56\left(\frac{\left|Y_{d s} Y_{s d}^{*}\right|}{M_{H}^{2}}\right) \mathrm{GeV}
$$

where we have assumed that $B_{K}^{\prime}=1$. From (67) we obtain a lower bound for $M_{H}: M_{H} \gtrsim$ $1.3 \times 10^{4} \sqrt{\left|Y_{d s} Y_{s d}^{*}\right|} \mathrm{TeV}$. Therefore, if $\left|Y_{d s}\right|,\left|Y_{s d}\right| \simeq \sqrt{m_{s} m_{d}} / v \sim 10^{-4}(v=246 \mathrm{GeV})$ is 
satisfied, then the lower bound reduces to $\sim O(1) \mathrm{TeV}$. Cheng and Sher showed that this is true in more general cases: If the Yukawa couplings satisfy $(\mathbf{Y})_{i j} \sim \sqrt{m_{i} m_{j}} / v$, then the lower bound of the mass of flavor changing neutral Higgs bosons is $O$ (few) $\mathrm{TeV}$.

We will show that in the $Q_{6}$ model this mechanism is partially embedded. To this end, we consider the Yukawa matrices (49) and find:

$$
\begin{aligned}
& \mathbf{Y}^{u 1} \simeq\left(\begin{array}{ccc}
0 & 0 & 0 \\
0 & 0 & 0.05 \sqrt{m_{c} m_{t}} \\
0 & 17 \sqrt{m_{c} m_{t}} & 0
\end{array}\right) \frac{\sqrt{2}}{v s_{\gamma^{u}} s_{\beta}}, \mathbf{Y}^{u 2} \simeq\left(\begin{array}{ccc}
0 & 0 & 1.3 \sqrt{m_{u} m_{t}} \\
0 & 0 & 0 \\
-408 \sqrt{m_{u} m_{t}} & 0 & 0
\end{array}\right) \frac{\sqrt{2}}{v s_{\gamma^{u}} s_{\beta}}, \\
& \mathbf{Y}^{u 3} \simeq\left(\begin{array}{ccc}
0 & 12 \sqrt{m_{u} m_{c}} & 0 \\
12 \sqrt{m_{u} m_{c}} & 0 & 0 \\
0 & 0 & 0.007 \sqrt{m_{t} m_{t}}
\end{array}\right) \frac{\sqrt{2}}{v c_{\gamma^{u}} s_{\beta}}, \\
& \mathbf{Y}^{d 1} \simeq\left(\begin{array}{ccc}
0 & 0 & 0 \\
0 & 0 & 0.3 \sqrt{m_{s} m_{b}} \\
0 & 2.9 \sqrt{m_{s} m_{b}} & 0
\end{array}\right) \frac{\sqrt{2}}{v s_{\gamma^{d}} c_{\beta}}, \mathbf{Y}^{d 2} \simeq\left(\begin{array}{ccc}
0 & 0 & 1.5 \sqrt{m_{d} m_{b}} \\
0 & 0 & 0 \\
-13 \sqrt{m_{d} m_{b}} & 0 & 0
\end{array}\right) \frac{\sqrt{2}}{v s_{\gamma^{d}} c_{\beta}}, \\
& \mathbf{Y}^{d 3} \simeq\left(\begin{array}{ccc}
0 & 1.0 \sqrt{m_{d} m_{s}} & 0 \\
1.0 \sqrt{m_{d} m_{s}} & 0 & 0 \\
0 & 0 & 0.9 \sqrt{m_{b} m_{b}}
\end{array}\right) \frac{\sqrt{2}}{v c_{\gamma^{d}} c_{\beta}},
\end{aligned}
$$

where we have used the central values of the parameters given in (57). The large $(3,2)$ and $(3,1)$ elements of $\mathbf{Y}^{u 1}$ and $\mathbf{Y}^{u 2}$, respectively, can induce flavor changing decay of the top quark, $t \rightarrow c+\bar{q}+q$ and $t \rightarrow u+\bar{q}+q$. However, the rate is very small. The large $(1,2)$ and $(2,1)$ elements of $\mathbf{Y}^{u 3}$ contribute dominantly to the mass deference of the neutral $D$ meson $\Delta M_{D} \simeq 1.4 \times 10^{-14} \mathrm{GeV}$. From a similar estimate that we have done above we find

$$
M_{H} \gtrsim \frac{\sqrt{2 \cdot 12 \cdot 12 m_{u} m_{c}}}{v c_{\gamma^{u}} s_{\beta}} \times 2.1 \times 10^{3} \mathrm{TeV} \simeq \frac{8.3}{c_{\gamma^{u}} s_{\beta}} \mathrm{TeV} .
$$

Here we have demonstrated that the Cheng-Sher mechanism is partially working in the $Q_{6}$ model. In the lepton sector one can do the same calculations, but it turned out that the constraints on the lower bound on $M_{H}$ are much weaker than (70) (see for instance [69]).

\section{B. FCNCs and CP violations from the soft supersymmetry breaking (SSB)}

We work in the super CKM basis and use mass insertion parameters $\left(\delta_{i j}^{\alpha}\right)_{L L, R R, L R}(\alpha=$ $u, d, e)$ to parameterize FCNCs and CP violations coming from the SSB sector. We start with the mass eigenstates of the quarks $u_{i L} \rightarrow u_{i L}^{\prime}=\left(U_{u L}\right)_{i j} u_{j L}, u_{i R}^{c} \rightarrow u_{i R}^{c \prime}=\left(U_{u R}\right)_{i j}^{*} u_{j R}^{c}$, where $u_{1}^{\prime}=u, u_{2}^{\prime}=c, u_{3}^{\prime}=t$, and similarly for the other matter fermions. Then we go to the super CKM basis for the matter fermions: $\tilde{u}_{i L} \rightarrow \tilde{u}_{i L}^{s}=\left(U_{u L}\right)_{i j} \tilde{u}_{j L}, \tilde{u}_{i R} \rightarrow \tilde{u}_{i R}^{s}=\left(U_{u R}\right)_{i j} \tilde{u}_{j R}$, etc. In the super CKM basis, the squark mass matrices become

$$
\tilde{\mathbf{M}}_{u}^{s 2}=\left(\begin{array}{cc}
m_{\tilde{u}_{i} L} m_{\tilde{u}_{j} L}\left[\mathbf{1}_{i j}+\left(\delta_{i j}^{u}\right)_{L L}\right] & \left(\Delta_{i i}^{u}\right)_{L R} \mathbf{1}_{i l}+m_{\tilde{u}_{i} L} m_{\tilde{u}_{l} R}\left(\delta_{i l}^{u}\right)_{L R} \\
\left(\Delta_{k k}^{u}\right)_{L R}^{*} \mathbf{1}_{k j}+m_{\tilde{u}_{k} L} m_{\tilde{u}_{j} R}\left(\delta_{j k}^{* u}\right)_{L R} & m_{\tilde{u}_{k} R} m_{\tilde{u}_{l} R}\left[\mathbf{1}_{k l}+\left(\delta_{k l}^{u}\right)_{R R}\right]
\end{array}\right),
$$

and

$$
\tilde{\mathbf{M}}_{d}^{s 2}=\left(\begin{array}{cc}
m_{\tilde{d}_{i} L} m_{\tilde{d}_{j} L}\left[\mathbf{1}_{i j}+\left(\delta_{i j}^{d}\right)_{L L}\right] & \left(\Delta_{i i}^{d}\right)_{L R} \mathbf{1}_{i l}+m_{\tilde{d}_{i} L} m_{\tilde{d}_{l} R}\left(\delta_{i l}^{d}\right)_{L R} \\
\left(\Delta_{k k}^{d}\right)_{L R}^{*} \mathbf{1}_{k j}+m_{\tilde{d}_{k} L} m_{\tilde{d}_{j} R}\left(\delta_{j k}^{* d}\right)_{L R} & m_{\tilde{d}_{k} R} m_{\tilde{d}_{l} R}\left[\mathbf{1}_{k l}+\left(\delta_{k l}^{d}\right)_{R R}\right]
\end{array}\right),
$$


respectively, where [32]

$$
\begin{aligned}
m_{u_{i}} \mathbf{1}_{i j} & =\left(U_{u L}^{\dagger} \mathbf{m}_{u} U_{u R}\right)_{i j}, m_{d_{i}} \mathbf{1}_{i j}=\left(U_{d L}^{\dagger} \mathbf{m}_{d} U_{d R}\right)_{i j} \\
m_{\tilde{u}_{i} L(R)}^{2} & =\left(U_{u L(R)}^{\dagger} \tilde{\mathbf{m}}_{Q(u)}^{2} U_{u L(R)}\right)_{i i}+m_{u_{i}}^{2}+c_{L(R)}^{u} M_{Z}^{2} \\
m_{\tilde{d}_{i} L(R)}^{2} & =\left(U_{d L(R)}^{\dagger} \tilde{\mathbf{m}}_{Q(d)}^{2} U_{d L(R)}\right)_{i i}+m_{d_{i}}^{2}+c_{L(R)}^{d} M_{Z}^{2} \\
\left(\delta_{i j}^{u(d)}\right)_{L L} & =\left[\left(U_{u(d) L}^{\dagger} \tilde{\mathbf{m}}_{Q}^{2} U_{u(d) L}\right)_{i j}-\left(U_{u(d) L}^{\dagger} \tilde{\mathbf{m}}_{Q}^{2} U_{u(d) L}\right)_{i i} \mathbf{1}_{i j}\right] / m_{\tilde{u}_{i}\left(\tilde{d}_{i}\right) L} m_{\tilde{u}_{j}\left(\tilde{d}_{j}\right) L}, \\
\left(\delta_{i j}^{u(d)}\right)_{R R} & =\left[\left(U_{u(d) R}^{\dagger} \tilde{\mathbf{m}}_{u(d)}^{2} U_{u(d) R}\right)_{i j}-\left(U_{u(d) R}^{\dagger} \tilde{\mathbf{m}}_{u(d)}^{2} U_{u(d) R}\right)_{i i} \mathbf{1}_{i j}\right] / m_{\tilde{u}_{i}\left(\tilde{d}_{i}\right) R} m_{\tilde{u}_{j}\left(\tilde{d}_{j}\right) R}, \\
\left(\Delta_{i j}^{u}\right)_{L R} & =\left(U_{u L}^{\dagger}\right)_{i k}\left\{-\mu_{I J}\left(Y_{k l}^{u I}\right)^{*}<h_{J}^{d 0}>+A_{k l}^{* u}\left(\mathbf{m}_{u}\right)_{k l}\right\}\left(U_{u R}\right)_{l j}, \\
\left(\Delta_{i j}^{d}\right)_{L R} & =\left(U_{d L}^{\dagger}\right)_{i k}\left\{\mu_{J I}\left(Y_{k l}^{d I}\right)<h_{J}^{u 0}>+A_{k l}^{* d}\left(\mathbf{m}_{d}\right)_{k l}\right\}\left(U_{d R}\right)_{l j}, \\
\left(\delta_{i j}^{u(d)}\right)_{L R} & =\left[\left(\Delta_{i j}^{u(d)}\right)_{L R}-\left(\Delta_{i i}^{u(d)}\right)_{L R} \mathbf{1}_{i j}\right] / m_{\tilde{u}_{i}\left(\tilde{d}_{i}\right) L} m_{\tilde{u}_{j}\left(\tilde{d}_{j}\right) R} .
\end{aligned}
$$

So far no assumption on flavor symmetry is made, and the squark mass matrices (71) and (72) cover the case of more than one pairs of Higgs doublet supermultiplets.

If three generations of a family are put into a one-dimensional and two-dimensional irreps of any group, then the soft scalar mass matrix for the sfermions has always a diagonal form:

$$
\tilde{\mathbf{m}}_{Q, L}^{2}=m_{\tilde{q}, \tilde{\ell}}^{2} \operatorname{diag} .\left(a_{L}^{q, \ell}, a_{L}^{q, \ell}, b_{L}^{q, \ell}\right), \tilde{\mathbf{m}}_{\alpha}^{2}=m_{\tilde{q}, \tilde{\ell}}^{2} \operatorname{diag} .\left(a_{R}^{\alpha}, a_{R}^{\alpha}, b_{R}^{\alpha}\right) \quad(\alpha=u, d, e),
$$

where $m_{\tilde{q}, \tilde{\ell}}$ denote the average of the squark and slepton masses, respectively, and $\left(a_{L(R)}, b_{L(R)}\right)$ are dimensionless free real parameters of $O(1)$. Further, flavor symmetry imposes the soft trilinear coupling matrices $(A$ terms $)$ to have the same structure as the Yukawa coupling matrices. They are real, because we impose CP invariance at the Lagrangian level. The imaginary parts of $\delta_{i j}^{\alpha}$ and $\Delta_{i j}^{\alpha}$ contribute to CP violating processes. Recall that the soft scalar mass matrices are real, because they are diagonal. The unitary matrices $U_{u L}$, etc. are complex, and so $\left(\delta_{i j}^{\alpha}\right)_{L L, R R}$ are complex in general. Note that the unitary matrices have the form $U=R P O$ in the case of $\mathcal{P}_{I}$ and $U=P R O$ in the case of $\mathcal{P}_{I I}$ (see (53) and (56) ), respectively, where only the phase matrices $P$ are complex. Since all the soft scalar mass matrices are diagonal with the structure $\tilde{\mathbf{m}}^{2} \sim \operatorname{diag} .(a, a, b)$, they commute with $P$ and $R$, so that $\left(\delta_{i j}^{\alpha}\right)_{L L, R R}$ have no imaginary part. Further, the phase of the $A$ term contribution to $\left(\Delta_{i j}^{\alpha}\right)_{L R}$ in (75) comes from the complex VEVs (see (29)), because CP is only spontaneously broken. Therefore, the $A$ term contribution has the same phase structure as the corresponding fermion mass matrix $\mathbf{m}_{\alpha}$. That is, defining the $A$ parameters as $h_{\gamma}^{\alpha}=A_{\gamma}^{\alpha} Y_{\gamma}^{\alpha} \quad\left(\alpha=u, d, e, \gamma=a, b, b^{\prime}, c\right)$, where $h_{\gamma}^{\alpha}$ are the soft tri-linear couplings, and the Yukawa couplings are defined in (49)-(51), we find that, except for $\left(\Delta_{i j}^{e}\right)_{L R}$ in the case of $\mathcal{P}_{I I}$, the $A$ term contributions to $\left(\Delta_{i j}^{\alpha}\right)_{L R}$ are real, too. Consequently, there is no $\mathrm{CP}$ violation originating from the SSB sector. Only the $\mu$ term contributions to $\left(\Delta_{i j}^{\alpha}\right)_{L R}$ are complex. The stringent constraints coming from the EDMs [34, 70] are automatically satisfied in this way of phase alignment, except for the $\mu$ term contributions. To suppress the contribution to the EDMs, therefore, one should have relatively small $\mu$ 's [44]. This is general if there are more than one pair of Higgs doublet supermultiplets.

The theoretical values of $\left(\delta_{i j}^{\alpha}\right)_{L L, R R, L R}$ for the present model are calculated below ${ }^{5}$, where

$$
\Delta a_{L}^{q, \ell}=a_{L}^{q, \ell}-b_{L}^{q, \ell}, \Delta a_{R}^{\alpha}=a_{R}^{\alpha}-b_{R}^{\alpha}, \tilde{A}_{\gamma}^{\alpha}=A_{\gamma}^{\alpha} / m_{\tilde{q}, \tilde{\ell}} \quad\left(\alpha=u, d, e, \gamma=a, b, b^{\prime}, c\right),
$$

\footnotetext{
${ }^{5}$ We do not consider the RG running effects of the SSB parameters [71]. See also [72].
} 
are introduced, and we assume that $m_{\tilde{q}_{i} L, R} m_{\tilde{q}_{i} L, R}=\delta_{i j} m_{\tilde{q}}^{2}, m_{\tilde{\ell}_{i} L_{R}} m_{\tilde{\ell}_{i} L, R}=\delta_{i j} m_{\tilde{\ell}}^{2}$. In Table 2 , theoretical values of $\delta$ 's and their experimental bounds [34, 35] are summarized.

$$
\begin{aligned}
\operatorname{Im}\left(\delta_{11}^{d}\right)_{L R} & \simeq 10^{-4} \operatorname{Im}\left[\left(-0.20 c_{\phi^{d}}+i 1.0 s_{\phi^{d}}\right) \tilde{\mu}_{+}^{d}-\left(0.20 s_{\phi^{d}}+i 2.2 c_{\phi^{d}}\right) \tilde{\mu}_{-}^{d}+0.05 \tilde{\mu}_{3}^{d}\right] \tilde{m}_{\tilde{q}^{-}}(7,9) \\
\left(\delta_{12}^{d}\right)_{L R} & \simeq 10^{-5}\left[1.7\left(\tilde{A}_{a}^{d}-\tilde{A}_{b}^{d}-\tilde{A}_{b^{\prime}}^{d}+\tilde{A}_{c}^{d}\right)\right. \\
& \left.-\left(8.6 c_{\phi^{d}}+i 1.1 s_{\phi^{d}}\right) \tilde{\mu}_{+}^{d}-\left(8.6 s_{\phi^{d}}-i 1.1 c_{\phi^{d}}\right) \tilde{\mu}_{-}^{d}+1.0 \tilde{\mu}_{3}^{d}\right] \tilde{m}_{\tilde{q}}^{-1}, \\
\left(\delta_{21}^{d}\right)_{L R} & \simeq 10^{-4}\left[-0.16\left(\tilde{A}_{a}^{d}-\tilde{A}_{b^{\prime}}^{d}\right)+0.19\left(\tilde{A}_{b}^{d}-\tilde{A}_{c}^{d}\right)\right. \\
& \left.+\left(0.78 c_{\phi^{d}}-i 3.8 s_{\phi^{d}}\right) \tilde{\mu}_{+}^{d}+\left(0.78 s_{\phi^{d}}+i 3.8 c_{\phi^{d}}\right) \tilde{\mu}_{-}^{d}-1.1 \tilde{\mu}_{3}^{d}\right] \tilde{m}_{\tilde{q}}^{-1}, \\
\left(\delta_{13}^{d}\right)_{L R} & \simeq 10^{-5}\left[-4.2\left(\tilde{A}_{a}^{d}-\tilde{A}_{b}^{d}\right)\right. \\
& \left.-\left(4.4 c_{\phi^{d}}+i 2.8 s_{\phi^{d}}\right) \tilde{\mu}_{+}^{d}-\left(4.4 s_{\phi^{d}}-i 2.8 c_{\phi^{d}}\right) \tilde{\mu}_{-}^{d}+0.42 \tilde{\mu}_{3}^{d}\right] \tilde{m}_{\tilde{q}}^{-1}, \\
\left(\delta_{31}^{d}\right)_{L R} & \simeq 10^{-3}\left[-0.40\left(\tilde{A}_{a}^{d}-\tilde{A}_{b^{\prime}}^{d}\right)\right. \\
& \left.+\left(2.1 c_{\phi^{d}}-i 9.6 s_{\phi^{d}}\right) \tilde{\mu}_{+}^{d}+\left(2.1 s_{\phi^{d}}+i 9.6 c_{\phi^{d}}\right) \tilde{\mu}_{-}^{d}-0.004 \tilde{\mu}_{3}^{d}\right] \tilde{m}_{\tilde{q}}^{-1}, \\
\left(\delta_{23}^{d}\right)_{L R} & \simeq 10^{-4}\left[1.6 \tilde{A}_{a}^{d}-1.9 \tilde{A}_{b}^{d}+0.3 \tilde{A}_{c}^{b^{\prime}}\right. \\
& \left.+\left(1.8 c_{\phi^{d}}-i 0.06 s_{\phi^{d}}\right) \tilde{\mu}_{+}^{d}+\left(1.8 s_{\phi^{d}}+i 0.06 c_{\phi^{d}}\right) \tilde{\mu}_{-}^{d}+0.03 \tilde{\mu}_{3}^{d}\right] \tilde{m}_{\tilde{q}}^{-1}, \\
\left(\delta_{32}^{d}\right)_{L R} & \simeq 10^{-3}\left[-1.7\left(\tilde{A}_{a}^{d}-\tilde{A}_{b}^{d}\right)\right. \\
& \left.+\left(8.8 c_{\phi^{d}}+i 2.2 s_{\phi^{d}}\right) \tilde{\mu}_{+}^{d}+\left(8.8 s_{\phi^{d}}-i 2.2 c_{\phi^{d}}\right) \tilde{\mu}_{-}^{d}+0.02 \tilde{\mu}_{3}^{d}\right] \tilde{m}_{\tilde{q}}^{-1}, \\
\operatorname{Im}\left(\delta_{11}^{u}\right)_{L R} & \simeq 3.5 \times 10^{-4} \operatorname{Im}\left(\tilde{\mu}_{3}^{u}\right) \tilde{m}_{\tilde{q}}^{-1}, \\
\left(\delta_{12}^{u}\right)_{L R} & \simeq 10^{-3}\left[-0.03\left(\tilde{A}_{a}^{u}-\tilde{A}_{b^{\prime}}^{u}\right)+0.03\left(\tilde{A}_{b}^{u}-\tilde{A}_{c}^{u}\right)\right. \\
& \left.-\left(0.01 c_{\phi^{u}}-i 2.2 s_{\phi^{u}}\right) \tilde{\mu}_{+}^{u}-\left(0.01 s_{\phi^{u}}+i 2.2 c_{\phi^{u}}\right) \tilde{\mu}_{-}^{u}+0.6 \tilde{\mu}_{3}^{u}\right] \tilde{m}_{\tilde{q}}^{-1}, \\
\left(\delta_{21}^{u}\right)_{L R} & \simeq 10^{-3}\left[-0.03\left(\tilde{A}_{a}^{u}-\tilde{A}_{b^{\prime}}^{u}\right)+0.03\left(\tilde{A}_{b}^{u}-\tilde{A}_{c}^{u}\right)\right. \\
& \left.\left.+i 0.01 s_{\phi^{u}}\right) \tilde{\mu}_{+}^{u}+\left(0.92 s_{\phi^{u}}-i 0.01 c_{\phi^{u}}\right) \tilde{\mu}_{-}^{u}-1.6 \tilde{\mu}_{3}^{u}\right] \tilde{m}_{\tilde{q}}^{-1},
\end{aligned}
$$

where

$$
\begin{aligned}
\tilde{\mu}_{+}^{u} & =-\left(\frac{\mu_{11}}{m_{\tilde{q}}} \frac{c_{\phi^{d}} s_{\gamma^{d}}}{s_{\gamma^{u}}}+\sqrt{2} \frac{\operatorname{Re}\left(\mu_{13}\right)}{m_{\tilde{q}}} \frac{c_{\gamma^{d}}}{s_{\gamma^{u}}}\right) \tan ^{-1} \beta \\
\tilde{\mu}_{-}^{u} & =\left(\frac{\mu_{11}}{m_{\tilde{q}}} \frac{s_{\phi^{d}} s_{\gamma^{d}}}{s_{\gamma^{u}}}-\sqrt{2} \frac{\operatorname{Im}\left(\mu_{13}\right)}{m_{\tilde{q}}} \frac{c_{\gamma^{d}}}{s_{\gamma^{u}}}\right) \tan ^{-1} \beta \\
\tilde{\mu}_{3}^{u} & =-2 \frac{\operatorname{Re}\left(\mu_{31} e^{-i \phi^{d}}\right)}{m_{\tilde{q}}} \frac{s_{\gamma^{d}}}{s_{\gamma^{u}}} \tan ^{-1} \beta, \tilde{\mu}_{3}^{d}=2 \frac{\operatorname{Re}\left(\mu_{13} e^{-i \phi^{u}}\right)}{m_{\tilde{q}}} \frac{s_{\gamma^{u}}}{s_{\gamma^{d}}} \tan \beta \\
\tilde{\mu}_{+}^{d} & =\left(\frac{\mu_{11}}{m_{\tilde{q}}} \frac{c_{\phi^{u}} s_{\gamma^{u}}}{s_{\gamma^{d}}}+\sqrt{2} \frac{\operatorname{Re}\left(\mu_{31}\right)}{m_{\tilde{q}}} \frac{c_{\gamma^{u}}}{s_{\gamma^{d}}}\right) \tan \beta, \tilde{\mu}_{-}^{d}=\left(-\frac{\mu_{11}}{m_{\tilde{q}}} \frac{s_{\phi^{u}} s_{\gamma^{u}}}{s_{\gamma^{d}}}+\sqrt{2} \frac{\operatorname{Im}\left(\mu_{31}\right)}{m_{\tilde{q}}} \frac{c_{\gamma^{u}}}{s_{\gamma^{d}}}\right) \tan \beta
\end{aligned}
$$

and the angles $s_{\gamma^{d}}$, etc. are defined in (32).. The $\mu$ term contributions above are complex and so contribute to EDMs. We see from from (79) and (83) that the strong constraint $\left|\operatorname{Im}\left(\delta_{11}^{u, d}\right)_{L R}^{\prime}\right| \lesssim 10^{-6}$ from the neutron EDM [34, 70] can be satisfied if $\mu$ 's are relatively small compared with the soft scalar masses [44, 45]. In contrast to this, $\left(\delta_{23}^{d}\right)_{L R}$ and $\left(\delta_{32}^{d}\right)_{L R}$, 
which contribute to $b \rightarrow s+\gamma$, are well below the upper limit $10^{-2}\left(500 \mathrm{GeV} / m_{\tilde{q}}\right)^{2}$. Further, the imaginary part of $\left(\delta_{23}^{d}\right)_{L R}$ and $\left(\delta_{32}^{d}\right)_{L R}$ contributes to the CP violation in the $B_{s}^{0}-\bar{B}_{s}^{0}$ mixing. Their size is small compared with $\left(\delta_{23}^{d}\right)_{L L, R R}$ given in Table 2, which however do not contribute to $\mathrm{CP}$ violation because they are real at the tree-level. At the one-loop level they can be complex, and the size can be as large as the tree-level size under certain circumstances [45]. With this (fine-tuned) one-loop contribution the theoretical value of the dimuon asymmetry can become close [45] to the order of the D0 measurement [73]. This large $\mathrm{CP}$ violation has been toned down by $\mathrm{CDF}$ [74] and than by the resent measurement at $\mathrm{LHCb}$ [75] (see also [60]). Therefore, that fine tuning is no longer necessary. Another interesting observation can be made on flavor violating decays of the top quark, i.e. $t \rightarrow u(c)+g$. From $\left(\delta_{13,31,23,32}^{u}\right)_{L R}$, we find the ratio $B(t \rightarrow u+g) / B(t \rightarrow c+g) \sim 10^{-4}$, which is consistent with the bound recently found by ATLAS [78].

\begin{tabular}{|c||c|c|}
\hline & Exp. bound & $Q_{6}$ Model $\left(\mathcal{P}_{I I}\right)$ \\
\hline \hline$\sqrt{\left|\operatorname{Re}\left(\delta_{12}^{d}\right)_{L L, R R}^{2}\right|}$ & $4.0 \times 10^{-2} \tilde{m}_{\tilde{q}}$ & $(L L) 4.0 \times 10^{-4} \Delta a_{L}^{q},(R R) 3.3 \times 10^{-2} \Delta a_{R}^{d}$ \\
\hline$\sqrt{\left|\operatorname{Re}\left(\delta_{12}^{d}\right)_{L L}\left(\delta_{12}^{d}\right)_{R R}\right|}$ & $2.8 \times 10^{-3} \tilde{m}_{\tilde{q}}$ & $3.6 \times 10^{-3} \sqrt{\Delta a_{L}^{q} \Delta a_{R}^{d}}$ \\
\hline$\sqrt{\left|\operatorname{Re}\left(\delta_{12}^{d}\right)_{L R}^{2}\right|}$ & $4.4 \times 10^{-3} \tilde{m}_{\tilde{q}}$ & Eq. (80) \\
\hline$\sqrt{\left|\operatorname{Re}\left(\delta_{13}^{d}\right)_{L L, R R}^{2}\right|}$ & $9.8 \times 10^{-2} \tilde{m}_{\tilde{q}}$ & $(L L) 1.0 \times 10^{-2} \Delta a_{L}^{q},(R R) 8.0 \times 10^{-2} \Delta a_{R}^{d}$ \\
\hline$\sqrt{\left|\operatorname{Re}\left(\delta_{13}^{d}\right)_{L L}\left(\delta_{13}^{d}\right)_{R R}\right|}$ & $1.8 \times 10^{-2} \tilde{m}_{\tilde{q}}$ & $2.8 \times 10^{-2} \sqrt{\Delta a_{L}^{q} \Delta a_{R}^{d}}$ \\
\hline$\sqrt{\left|\operatorname{Re}\left(\delta_{13}^{d}\right)_{L R}^{2}\right|}$ & $3.3 \times 10^{-2} \tilde{m}_{\tilde{q}}$ & Eq. (81) \\
\hline$\sqrt{\left|\operatorname{Re}\left(\delta_{12}^{u}\right)_{L L, R R}^{2}\right|}$ & $1.5 \times 10^{-1} \tilde{m}_{\tilde{q}}(L L) 2.9 \times 10^{-8} \Delta a_{L}^{q},(R R) 4.3 \times 10^{-1} \Delta a_{R}^{u}$ \\
\hline$\sqrt{\left|\operatorname{Re}\left(\delta_{12}^{u}\right)_{L L}\left(\delta_{12}^{u}\right)_{R R}\right|}$ & $1.7 \times 10^{-2} \tilde{m}_{\tilde{q}}$ & $1.1 \times 10^{-4} \sqrt{\Delta a_{L}^{q} \Delta a_{R}^{u}}$ \\
\hline$\sqrt{\left|\operatorname{Re}\left(\delta_{12}^{u}\right)_{L R}^{2}\right|}$ & $3.1 \times 10^{-2} \tilde{m}_{\tilde{q}}$ & Eq. (84) \\
\hline$\left|\left(\delta_{23}^{d}\right)_{L L, R R}\right|$ & $8.2 \tilde{m}_{\tilde{q}}^{2}$ & $(L L) 3.9 \times 10^{-2} \Delta a_{L}^{q},(R R) 3.5 \times 10^{-1} \Delta a_{R}^{d}$ \\
\hline$\left|\left(\delta_{23}^{d}\right)_{L R}\right|$ & $1.6 \times 10^{-2} \tilde{m}_{\tilde{q}}^{2}$ & Eq. (82) \\
\hline$\left|\operatorname{Im}\left(\delta_{11}^{d}\right)_{L R}\right|$ & $3.0 \times 10^{-6} \tilde{m}_{\tilde{q}}$ & Eq. (79) \\
\hline$\left|\operatorname{Im}\left(\delta_{11}^{u}\right)_{L R}\right|$ & $5.9 \times 10^{-6} \tilde{m}_{\tilde{q}}$ & Eq. (83) \\
\hline
\end{tabular}

TABLE II: Experimental bounds on $\left(\delta_{i j}^{u, d}\right)_{L L, R R, L R}$ 34, 35] and their theoretical values in the quark sector of the $Q_{6}$ model for $\mathcal{P}_{I I}$ (see also Kobayashi et al. in [40]), where $\tilde{m}_{\tilde{q}}$ denotes $m_{\tilde{q}} / 500 \mathrm{GeV}$, and $\Delta a_{L, R}$ and $\tilde{A}$ are given in (78).

Now we come to the lepton sector. As it is noticed, the phase alignment does not work for the lepton sector in the case of $\mathcal{P}_{I I}$. There are two phases, $\phi^{u}$ and $\phi^{d}$, that enter into the CKM and MNS matrices. One combination $\phi=\phi^{u}-\phi^{d}$ is basically fixed around 0.0838 (see (57)) to produce the correct Kobayashi-Maskawa CP phase. The other would be fixed if $\mathrm{CP}$ violation in the neutrino sector is precisely measured. Then the phase that enters into the soft mass insertions would be completely fixed. In other words we can express the mass insertions as a function of $\sin ^{2} \theta_{13}$, because in the case of $\mathcal{P}_{I I}$ the CP phase $\delta_{C P}$ and $\sin ^{2} \theta_{13}$ are intimately related. This is done in Fig. 4. We plot $\left|\left(\delta_{12}^{e}\right)_{L L}\right| / \Delta a_{L}^{\ell}$ and $\left|\left(\delta_{12}^{e}\right)_{R R}\right| / \Delta a_{L}^{\ell}$ against $\sin ^{2} \theta_{13}$, respectively. We see from Fig. 4 that $\left|\left(\delta_{12}^{e}\right)_{L L}\right| / \Delta a_{L}^{\ell}$ does not change very much as $\sin ^{2} \theta_{13}$ varies, while $\left|\left(\delta_{12}^{e}\right)_{R R}\right| / \Delta a_{R}^{\ell}$ depends significantly on $\sin ^{2} \theta_{13}$. 

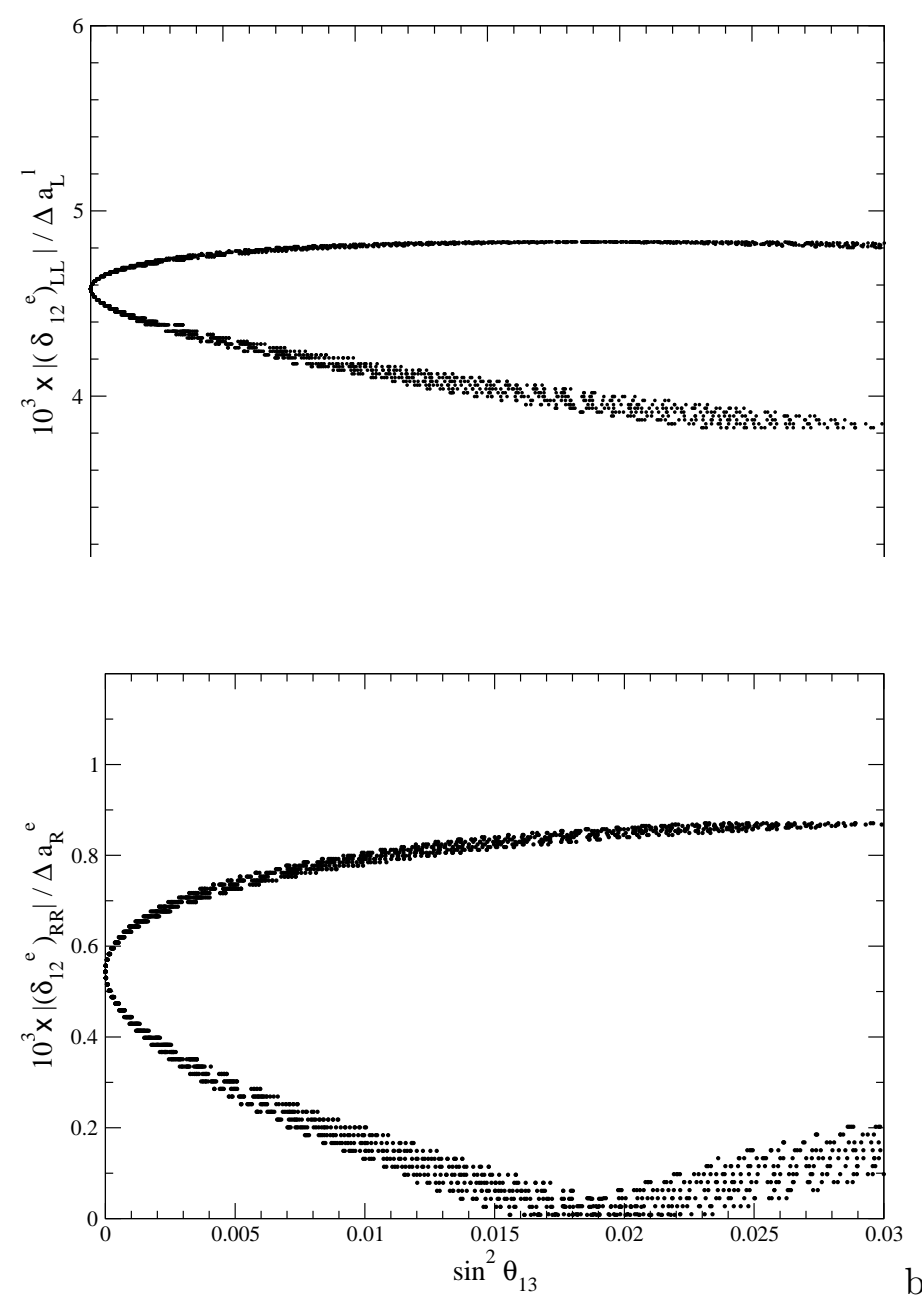

FIG. 4: $\left|\left(\delta_{12}^{e}\right)_{L L}\right| / \Delta a_{L}^{\ell}$ against $\sin ^{2} \theta_{13}$ (a) and $\left|\left(\delta_{12}^{e}\right)_{R R}\right| / \Delta a_{R}^{e}(\mathrm{~b})$. The parameter space is the same as for Fig. 2 ,

Unfortunately, there is no common range of $\sin ^{2} \theta_{13}$ such that the both insertions become small. The experimental bounds are [34, 35] ${ }^{6}$ :

$$
\left|\left(\delta_{12}^{e}\right)_{L L}\right| \lesssim 1.8 \times 10^{-4} \tilde{m}_{\tilde{\ell}}^{2},\left|\left(\delta_{12}^{e}\right)_{R R}\right| \lesssim 3.6 \times 10^{-4} \tilde{m}_{\tilde{\ell}}^{2},\left|\left(\delta_{12}^{e}\right)_{L R}\right| \lesssim 3.3 \times 10^{-6} \tilde{m}_{\tilde{\ell}}^{2},
$$

where $\tilde{m}_{\tilde{\ell}}=m_{\tilde{\ell}} / 300 \mathrm{GeV}$. In Fig. 5 we plot $\left|\left(\bar{\delta}_{12}^{e}\right)_{L R}\right|$ against $\sin \phi^{d}$ with $\phi$ fixed at 0.08375 , where we varied the dimensionless parameters $\tilde{A}_{\gamma}^{e}$ (defined in (178)) from -5 to 5 , and $\left|\left(\bar{\delta}_{12}^{e}\right)_{L R}\right|$ is defined as

$$
\left(\bar{\delta}_{12}^{e}\right)_{L R}=\left(\delta_{12}^{e}\right)_{L R} \tilde{m}_{\tilde{\ell}}^{-1} /\left(\sum_{\gamma=a, b, b^{\prime}, c}\left|\tilde{A}_{\gamma}^{e}\right|\right)
$$

We see from Fig. [5 that the size of $\left(\delta_{12}^{e}\right)_{L R}$ is of order of the experimental bound (866), and that it has no strong dependence of $\sin \phi^{d}$ and hence of $\sin ^{2} \theta_{13}$.

${ }_{6}$ The recent experimental bounds $B(\mu \rightarrow e+\gamma) \lesssim 2.4 \times 10^{-12}\left[76\right.$ and $B(\tau \rightarrow \mu(e)+\gamma) \lesssim 4.4(3.3) \times 10^{-8}$ [7] are included. 


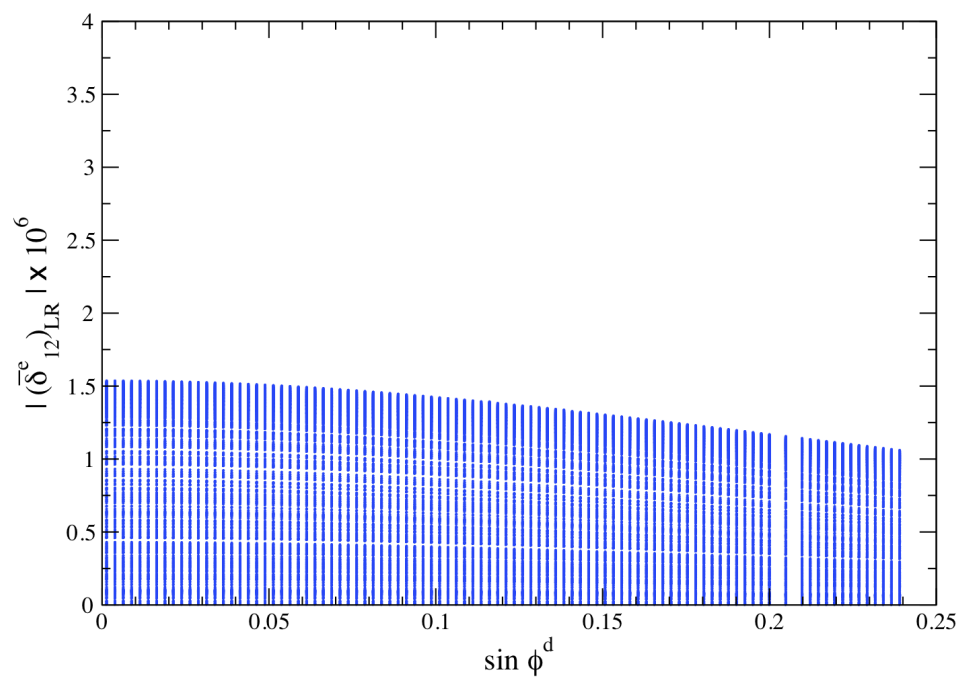

FIG. 5: $\left(\bar{\delta}_{12}^{e}\right)_{L R}$ against $\sin \phi^{d}$, where the dimensionless parameters $\tilde{A}_{\gamma}^{e}$ are varied from -5 to 5 . $\left(\bar{\delta}_{i j}^{e}\right)_{L R}$ is defined in (87).

We see thus from Tables 2 and Figs. 保d 5 that $Q_{6}$ flavor symmetry can well soften the FCNC problem of the SSB sector. The recent LHC data suggest that the superpartner masses are heavier than those assumed here [79, 80]. If this is the case the constraints are better satisfied.

\section{SUMMARY}

Obviously, the flavor problem is one of the most difficult problems. So far we do not have a magic bullet to solve this problem. Non-abelian flavor symmetry can soften the problem and therefore might give a hint to a solution. In this contribution I restricted myself to renormalizable models of flavor with a non-abelian discrete flavor symmetry, which is at most softly broken at some tera scale. This setting without supersymmetry may lead to problems because of FCNCs and of a fine tuning in the scalar potential. The fine tuning problem may be softened in supersymmetric models. In softly broken supersymmetric theories, the soft breaking terms of flavor symmetry can be classified, which I discussed in a very general form.

To point out basic features of supersymmetric models of flavor with multiple Higgs doublets I considered a specific model based on the finite flavor group $Q_{6}$ [7]. To make spontaneous breaking of $\mathrm{CP}$ possible, the Higgs sector was further extended [46] so as to include a certain set of SM singlet Higgs multiplets. With them the upper bound of the lightest Higgs mass upper bound can slightly increase. In this SM singlet Higgs sector, flavor symmetry and $\mathrm{CP}$ are spontaneously broken at a tera scale, which is very close to supersymmetry breaking scale. This may give a hint that flavor symmetry, CP and supersymmetry are broken within the same sector.

The $Q_{6}$ model of flavor yields interesting predictions for the CKM and MNS mixing 
parameters, which compare very well with experimental data. Large B-factories [81, 82] with more precise determination of quark masses [83] will be able to test the predictions in the quark sector [84]. In the lepton sector, the predictions for the ground state $\mathcal{P}_{I I}$ are particularly interesting, because the neutrino mass hierarchy is inverted with nearly maximal $\mathrm{CP}$ violation along with nearly maximal mixing of atmospheric neutrinos: The CP phase, the average neutrino mass $\left\langle m_{e e}\right\rangle$, and $\sin ^{2} \theta_{23}$ can be expressed as a function of $\sin ^{2} \theta_{13}$. These predictions can also be tested by the future neutrino experiments (see [67] and ([68]).

Non-abelian flavor symmetry can not only relate the fermion masses and mixing parameters, but also suppress FCNCs. Flavor symmetry embeds the Cheng-Sher mechanism automatically, which suppresses the tree-level FCNCs, making the flavor symmetry scale decrease down to tera scale, and suppress FCNCs coming from the soft breaking of supersymmetry as well. The CP problem of softly broken supersymmetric theories is solved by virtue of spontaneous CP violation. This implies that the soft supersymmetry breaking parameters such as the gluino mass are all real. The phase of the trilinear soft breaking $A$ terms, since they arise spontaneously, will align with the phases in the fermion mass matrices. A self-alignment mechanism is going on. Thus the $A$ terms do not generate $\mathrm{CP}$ violation. Thanks to flavor symmetry, the mass insertion parameters $\left(\delta_{i j}^{\alpha}\right)_{L L, R R, L R}$ depend on only few independent parameters. Their ratio, e.g., $\left(\delta_{12}^{e}\right)_{L L} /\left(\delta_{13}^{e}\right)_{R R}$, is fixed, which is a unique prediction. Therefore, flavor violations are controlled by flavor symmetry. Thus new observations of flavor and $\mathrm{CP}$ violations can also test flavor symmetry at tera scale [85].

\section{Acknowledgments}

I thank Jose Valle, Stefano Morisi and Claudia Hagedorn for inviting me to a contribution to the special issue on "Flavor Symmetries and Neutrino Oscillations". I also thank T. Araki, K.S. Babu, E. Itou, Y. Kaburaki, Y. Kajiyama, K. Kawashima, N. Kifune, T. Kobayashi, K. Konya, A. Lenz, A. Mondragon, M. Mondragon, E. Rodriguez-Jauregui, H. Okada, F. Sakamaki, H. Terao for exiting collaborations. Many results presented here have been obtained with them. The work of JK is partially supported by a Grant-in-Aid for Scientific Research (C) from Japan Society for the Promotion of Science (No. 22540271).

[1] See for a recent review: G. Altarelli and F. Feruglio, Rev. Mod. Phys. 82 (2010) 2701 arXiv:1002.0211 [hep-ph]].

[2] S. Pakvasa and H. Sugawara, Phys. Lett. B 73 (1978) 61; T. Brown, N. Deshpande, S. Pakvasa and H. Sugawara, Phys. Lett. B 141 (1984) 95.

[3] E. Ma, Phys. Rev. D 43 (1991) 2761; Phys. Rev. D 44 (1991) 587; P. H. Frampton and T. W. Kephart, Int. J. Mod. Phys. A 10 (1995) 4689 arXiv:hep-ph/9409330; P. H. Frampton and O. C. W. Kong, Phys. Rev. Lett. 75 (1995) 781 arXiv:hep-ph/9502395; Phys. Rev. D 53 (1996) 2293 arXiv:hep-ph/9511343; P. H. Frampton and A. Rasin, Phys. Lett. B 478 (2000) 424 arXiv:hep-ph/9910522]; W. Grimus and L. Lavoura, JHEP 0107 (2001) 045 arXiv:hep-ph/0105212.

[4] E. Ma and G. Rajasekaran, Phys. Rev. D 64 (2001) 113012 arXiv:hep-ph/0106291]; E. Ma, Mod. Phys. Lett. A 17 (2002) 627 arXiv:hep-ph/0203238; K. S. Babu, 
E. Ma and J. W. F. Valle, Phys. Lett. B 552 (2003) 207 hep-ph/0206292]; G. Seidl, arXiv:hep-ph/0301044.

[5] J. Kubo, A. Mondragon, M. Mondragon and E. Rodriguez-Jauregui, Prog. Theor. Phys. 109 (2003) 795 [Erratum-ibid. 114 (2005) 287] arXiv:hep-ph/0302196]; J. Kubo, Phys. Lett. B 578 (2004) 156 [Erratum-ibid. B 619 (2005) 387] arXiv:hep-ph/0309167].

[6] W. Grimus and L. Lavoura, Phys. Lett. B 572 (2003) 189 arXiv:hep-ph/0305046]; W. Grimus, A. S. Joshipura, S. Kaneko, L. Lavoura and M. Tanimoto, JHEP 0407 (2004) 078 arXiv:hep-ph/0407112]; W. Grimus, A. S. Joshipura, S. Kaneko, L. Lavoura, H. Sawanaka and M. Tanimoto, Nucl. Phys. B 713 (2005) 151 arXiv:hep-ph/0408123]; M. Frigerio, S. Kaneko, E. Ma and M. Tanimoto, Phys. Rev. D 71 (2005) 011901 [arXiv:hep-ph/0409187].

[7] K. S. Babu and J. Kubo, Phys. Rev. D 71 (2005) 056006 arXiv:hep-ph/0411226.

[8] L. Lavoura and E. Ma, Mod. Phys. Lett. A 20 (2005) 1217 [arXiv:hep-ph/0502181]; C. Hagedorn and W. Rodejohann, JHEP 0507 (2005) 034 [arXiv:hep-ph/0503143]; W. Grimus and L. Lavoura, JHEP 0508 (2005) 013 arXiv:hep-ph/0504153]; G. Altarelli and F. Feruglio, Nucl. Phys. B 720 (2005) 64 arXiv:hep-ph/0504165.

[9] S. L. Chen, M. Frigerio and E. Ma, Nucl. Phys. B 724 (2005) 423 arXiv:hep-ph/0504181]; S. L. Chen and E. Ma, Phys. Lett. B 620 (2005) 151 [arXiv:hep-ph/0505064].

[10] M. Hirsch, A. Villanova del Moral, J. W. F. Valle and E. Ma, Phys. Rev. D 72 (2005) 091301 [Erratum-ibid. D 72 (2005) 119904] arXiv:hep-ph/0507148]; I. de Medeiros Varzielas and G. G. Ross, Nucl. Phys. B 733 (2006) 31 arXiv:hep-ph/0507176]; K. S. Babu and X. G. He, arXiv:hep-ph/0507217; W. Grimus and L. Lavoura, JHEP 0601 (2006) 018 arXiv:hep-ph/0509239; F. Caravaglios and S. Morisi, arXiv:hep-ph/0510321; I. de Medeiros Varzielas, S. F. King and G. G. Ross, Phys. Lett. B 644 (2007) 153 arXiv:hep-ph/0512313; arXiv:hep-ph/0503234; T. Teshima, Phys. Rev. D 73 (2006) 045019 arXiv:hep-ph/0509094; N. Haba and K. Yoshioka, Nucl. Phys. B 739 (2006) 254 [arXiv:hep-ph/0511108].

[11] Y. Kajiyama, E. Itou and J. Kubo, Nucl. Phys. B 743 (2006) 74 arXiv:hep-ph/0511268.

[12] C. Hagedorn, M. Lindner and R. N. Mohapatra, JHEP 0606 (2006) 042 arXiv:hep-ph/0602244; C. Hagedorn, M. Lindner and F. Plentinger, Phys. Rev. D 74 (2006) 025007 arXiv:hep-ph/0604265]; E. Ma, H. Sawanaka and M. Tanimoto, Phys. Lett. B 641 (2006) 301 arXiv:hep-ph/0606103; S. F. King and M. Malinsky, Phys. Lett. B 645 (2007) 351 arXiv:hep-ph/0610250]; T. Kobayashi, H. P. Nilles, F. Ploger, S. Raby and M. Ratz, Nucl. Phys. B 768 (2007) 135 arXiv:hep-ph/0611020; Y. Kajiyama, JHEP 0704 (2007) 007 arXiv:hep-ph/0702056]; F. Feruglio, C. Hagedorn, Y. Lin and L. Merlo, Nucl. Phys. B 775 (2007) 120 arXiv:hep-ph/0702194; M. Hirsch, A. S. Joshipura, S. Kaneko and J. W. F. Valle, Phys. Rev. Lett. 99 (2007) 151802 arXiv:hep-ph/0703046; A. Blum, C. Hagedorn and M. Lindner, Phys. Rev. D 77 (2008) 076004 arXiv:0709.3450 [hep-ph]]; W. Grimus and H. Kuhbock, Phys. Rev. D 77 (2008) 055008 arXiv:0710.1585 [hep-ph]].

[13] R. Howl and S. F. King, JHEP 0805 (2008) 008 arXiv:0802.1909 [hep-ph]], G. Altarelli, F. Feruglio and C. Hagedorn, JHEP 0803 (2008) 052 [arXiv:0802.0090 [hep-ph]]; F. Feruglio, C. Hagedorn, Y. Lin and L. Merlo, Nucl. Phys. B 809 (2009) 218 [arXiv:0807.3160 [hep-ph]]; T. Kobayashi, Y. Omura and K. Yoshioka, Phys. Rev. D 78 (2008) 115006 arXiv:0809.3064 [hep-ph]]; C. S. Lam, Phys. Rev. D 78 (2008) 073015 arXiv:0809.1185 [hep-ph]]; F. Bazzocchi, M. Frigerio and S. Morisi, Phys. Rev. D 78 (2008) 116018 arXiv:0809.3573 [hep-ph]]; M. Hirsch, S. Morisi and J. W. F. Valle, Phys. Rev. D 79 (2009) 016001 arXiv:0810.0121 [hep-ph]]; C. Hagedorn, M. A. Schmidt and A. Y. Smirnov, Phys. Rev. D 79 (2009) 036002 arXiv:0811.2955 [hep-ph]]; L. L. Everett and A. J. Stuart, Phys. Rev. D 79 (2009) 085005 
arXiv:0812.1057 [hep-ph]]; H. Ishimori, Y. Shimizu and M. Tanimoto, Prog. Theor. Phys. 121 (2009) 769 [arXiv:0812.5031 [hep-ph]].

[14] F. Bazzocchi, L. Merlo and S. Morisi, Nucl. Phys. B 816 (2009) 204 [arXiv:0901.2086 [hep-ph]]; Phys. Rev. D 80 (2009) 053003 arXiv:0902.2849 [hep-ph]]; A. Blum and C. Hagedorn, Nucl. Phys. B 821 (2009) 327 arXiv:0902.4885 [hep-ph]]; G. Altarelli, F. Feruglio and L. Merlo, JHEP 0905 (2009) 020 arXiv:0903.1940 [hep-ph]]; S. F. King and C. Luhn, Nucl. Phys. B 820 (2009) 269 arXiv:0905.1686 [hep-ph]]; M. Hirsch, S. Morisi and J. W. F. Valle, Phys. Lett. B 679 (2009) 454 [arXiv:0905.3056 [hep-ph]]; A. Adulpravitchai, A. Blum and M. Lindner, JHEP 0907 (2009) 053 arXiv:0906.0468 [hep-ph]]; W. Grimus, L. Lavoura and P. O. Ludl, J. Phys. G 36 (2009) 115007 arXiv:0906.2689 [hep-ph]]; F. Feruglio, C. Hagedorn and L. Merlo, JHEP 1003 (2010) 084 arXiv:0910.4058 [hep-ph]]; S. Morisi and E. Peinado, Phys. Rev. D 80 (2009) 113011 arXiv:0910.4389 [hep-ph]]; S. F. King and C. Luhn, Nucl. Phys. B 832 (2010) 414 arXiv:0912.1344 [hep-ph]].

[15] K. S. Babu, J. C. Pati and Z. Tavartkiladze, JHEP 1006 (2010) 084 [arXiv:1003.2625] [hep-ph]]; R. de Adelhart Toorop, F. Bazzocchi and L. Merlo, JHEP 1008 (2010) 001 arXiv:1003.4502 [hep-ph]]; C. Hagedorn, S. F. King and C. Luhn, JHEP 1006 (2010) 048 [arXiv:1003.4249 [hepph]]; I. K. Cooper, S. F. King and C. Luhn, Phys. Lett. B 690 (2010) 396 arXiv:1004.3243 [hep-ph]]; Z. z. Xing, D. Yang and S. Zhou, Phys. Lett. B 690 (2010) 304 arXiv:1004.4234 [hep-ph]]; D. Meloni, S. Morisi and E. Peinado, J. Phys. G 38 (2011) 015003 arXiv:1005.3482 [hep-ph]]; K. S. Babu and S. Gabriel, Phys. Rev. D 82 (2010) 073014 arXiv:1006.0203 [hepph]]; G. Bhattacharyya, P. Leser and H. Pas, Phys. Rev. D 83 (2011) 011701 arXiv:1006.5597 [hep-ph]]; J. N. Esteves, F. R. Joaquim, A. S. Joshipura, J. C. Romao, M. A. Tortola and J. W. F. Valle, Phys. Rev. D 82 (2010) 073008 arXiv:1007.0898 [hep-ph]]. Y. Daikoku and H. Okada, arXiv:1008.0914 [hep-ph]; N. Haba, Y. Kajiyama, S. Matsumoto, H. Okada and K. Yoshioka, Phys. Lett. B 695 (2011) 476 arXiv:1008.4777 [hep-ph]]; M. K. Parida, P. K. Sahu and K. Bora, Phys. Rev. D 83 (2011) 093004 arXiv:1011.4577 [hep-ph]]; Phys. Lett. B 698 (2011) 131 arXiv:1011.4928 [hep-ph]]; Y. Kajiyama and H. Okada, Nucl. Phys. B 848 (2011) 303 arXiv:1011.5753 [hep-ph]].

[16] F. Gonzalez Canales and A. Mondragon, J. Phys. Conf. Ser. 287 (2011) 012015 arXiv:1101.3807 [hep-ph]]; T. Teshima and Y. Okumura, Phys. Rev. D 84 (2011) 016003 arXiv:1103.6127 [hep-ph]]; Y. Kajiyama, H. Okada and T. Toma, Eur. Phys. J. C 71 (2011) 1688 arXiv:1104.0367 [hep-ph]]; S. Zhou, Phys. Lett. B 704 (2011) 291 [arXiv:1106.4808 [hepph]]; M. S. Boucenna, M. Hirsch, S. Morisi, E. Peinado, M. Taoso and J. W. F. Valle, JHEP 1105 (2011) 037 arXiv:1101.2874 [hep-ph]]; Q. H. Cao, S. Khalil, E. Ma and H. Okada, Phys. Rev. D 84 (2011) 071302 arXiv:1108.0570 [hep-ph]]; G. J. Ding, L. L. Everett and A. J. Stuart, Nucl. Phys. B 857 (2012) 219 [arXiv:1110.1688 [hep-ph]]; K. Hashimoto and H. Okada, arXiv:1110.3640 [hep-ph]; I. d. M. Varzielas, JHEP 1201 (2012) 097 arXiv:1111.3952 [hepph]]; P. V. Dong, H. N. Long, C. H. Nam and V. V. Vien, arXiv:1111.6360 [hep-ph]; A. J. Buras, M. V. Carlucci, L. Merlo and E. Stamou, arXiv:1112.4477 [hep-ph]; T. Araki and Y. F. Li, arXiv:1112.5819 [hep-ph]; F. Bazzocchi, S. Morisi, E. Peinado, J. W. F. Valle and A. Vicente, arXiv:1202.1529 [hep-ph]; T. Teshima, arXiv:1202.4528 [hep-ph].

[17] Finite groups are comprehensively treated in: P. H. Frampton and T. W. Kephart, Phys. Rev. D 64 (2001) 086007 arXiv:hep-th/0011186]; H. Ishimori, T. Kobayashi, H. Ohki, Y. Shimizu, H. Okada and M. Tanimoto, Prog. Theor. Phys. Suppl. 183 (2010) 1 arXiv:1003.3552 [hepth]]; W. Grimus and P. O. Ludl, arXiv:1110.6376 [hep-ph].

[18] M. Kobayashi and T. Maskawa, Prog. Theor. Phys. 49 (1973) 652. 
[19] J. D. Bjorken and S. Weinberg, Phys. Rev. Lett. 38 (1977) 622; B. McWilliams and L. F. Li, Nucl. Phys. B 179 (1981) 62; O. U. Shanker, Nucl. Phys. B 206 (1982) 253.

[20] D. Atwood, L. Reina and A. Soni, Phys. Rev. D 55 (1997) 3156 arXiv:hep-ph/9609279; and see also: W. Grimus and L. Lavoura, Phys. Rev. D 66 (2002) 014016 arXiv:hep-ph/0204070.

[21] W. Grimus, L. Lavoura, O. M. Ogreid and P. Osland, J. Phys. G 35 (2008) 075001 arXiv:0711.4022 [hep-ph]].

[22] Two Higgs doublet models are extensively reviewed in: G. C. Branco, P. M. Ferreira, L. Lavoura, M. N. Rebelo, M. Sher and J. P. Silva, arXiv:1106.0034 [hep-ph].

[23] T. P. Cheng and M. Sher, Phys. Rev. D 35 (1987) 3484.

[24] J. Wess and B. Zumino, Phys. Lett. B 49 (1974) 52.

[25] J. Iliopoulos and B. Zumino, Nucl. Phys. B 76 (1974) 310; K. Fujikawa and W. Lang, Nucl. Phys. B 88 (1975) 61.

[26] The classic reviews on supersymmetric models are: H. P. Nilles, Phys. Rept. 110 (1984) 1; H. E. Haber, G. L. Kane, Phys. Rept. 117 (1985) 75-263; S. P. Martin, arXiv:hep-ph/9709356 D. J. H. Chung, L. L. Everett, G. L. Kane, S. F. King, J. D. Lykken and L. T. Wang, Phys. Rept. 407 (2005) 1 arXiv:hep-ph/0312378.

[27] S. Ferrara, L. Girardello and F. Palumbo, Phys. Rev. D 20 (1979) 403; K. Harada and N. Sakai, Prog. Theor. Phys. 67 (1982) 1877; L. Girardello and M. T. Grisaru, Nucl. Phys. B 194 (1982) 65.

[28] I. Jack and D. R. T. Jones, Phys. Lett. B 415 (1997) 383 arXiv:hep-ph/9709364; L. V. Avdeev, D. I. Kazakov and I. N. Kondrashuk, Nucl. Phys. B 510 (1998) 289 arXiv:hep-ph/9709397]; I. Jack, D. R. T. Jones and A. Pickering, Phys. Lett. B 426 (1998) 73 arXiv:hep-ph/9712542.

[29] K. Symanzik, in Fundamental Interactions at High-Energies, ed. by A. Mutter et al. (Gordon and Breach, 1970).

[30] J. R. Ellis and D. V. Nanopoulos, Phys. Lett. B 110 (1982) 44; R. Barbieri and R. Gatto, Phys. Lett. B 110 (1982) 211; B. A. Campbell, Phys. Rev. D 28 (1983) 209; M. J. Duncan, Nucl. Phys. B 221 (1983) 285; J. F. Donoghue, H. P. Nilles and D. Wyler, Phys. Lett. B 128 (1983) 55; J. M. Gerard, W. Grimus, A. Raychaudhuri and G. Zoupanos, Phys. Lett. B 140 (1984) 349.

[31] J. R. Ellis, S. Ferrara and D. V. Nanopoulos, Phys. Lett. B 114 (1982) 231; W. Buchmuller and D. Wyler, Phys. Lett. B 121 (1983) 321; J. Polchinski and M. B. Wise, Phys. Lett. B 125 (1983) 393; F. del Aguila, M. B. Gavela, J. A. Grifols and A. Mendez, Phys. Lett. B 126 (1983) 71 [Erratum-ibid. B 129 (1983) 473]; D. V. Nanopoulos and M. Srednicki, Phys. Lett. B 128 (1983) 61.

[32] L. J. Hall, V. A. Kostelecky and S. Raby, Nucl. Phys. B 267 (1986) 415; F. Gabbiani and A. Masiero, Nucl. Phys. B 322 (1989) 235.

[33] S. Bertolini, F. Borzumati, A. Masiero and G. Ridolfi, Nucl. Phys. B 353 (1991) 591; R. Barbieri and G. F. Giudice, Phys. Lett. B 309 (1993) 86 arXiv:hep-ph/9303270; G. C. Branco, G. C. Cho, Y. Kizukuri and N. Oshimo, Phys. Lett. B 337 (1994) 316 arXiv:hep-ph/9408229].

[34] F. Gabbiani, E. Gabrielli, A. Masiero and L. Silvestrini, Nucl. Phys. B 477 (1996) 321 arXiv:hep-ph/9604387.

[35] M. Misiak, S. Pokorski and J. Rosiek, Adv. Ser. Direct. High Energy Phys. 15 (1998) 795 arXiv:hep-ph/9703442]; L. Silvestrini, Int. J. Mod. Phys. A 21 (2006) 1738 arXiv:hep-ph/0510077; M. Ciuchini and L. Silvestrini, Phys. Rev. Lett. 97 (2006) 021803 arXiv:hep-ph/0603114; M. Ciuchini, E. Franco, D. Guadagnoli, V. Lubicz, M. Pierini, 
V. Porretti and L. Silvestrini, arXiv:hep-ph/0703204; E. Golowich, J. Hewett, S. Pakvasa and A. A. Petrov, arXiv:0705.3650 [hep-ph]; J. Hisano, Nucl. Phys. Proc. Suppl. 137 (2004) 169 arXiv:hep-ph/0405185.

[36] M. Dine, R. G. Leigh and A. Kagan, Phys. Rev. D 48 (1993) 4269 arXiv:hep-ph/9304299; R. Barbieri, G. R. Dvali and L. J. Hall, Phys. Lett. B 377 (1996) 76 arXiv:hep-ph/9512388; M. C. Chen and K. T. Mahanthappa, Phys. Rev. D 65 (2002) 053010 arXiv:hep-ph/0106093.

[37] K. S. Babu and S. M. Barr, Phys. Lett. B 387 (1996) 87 [arXiv:hep-ph/9606384]; K. S. Babu and R. N. Mohapatra, Phys. Rev. Lett. 83 (1999) 2522 arXiv:hep-ph/9906271.

[38] P. Pouliot and N. Seiberg, Phys. Lett. B 318 (1993) 169 arXiv:hep-ph/9308363; D. B. Kaplan and M. Schmaltz, Phys. Rev. D 49 (1994) 3741 arXiv:hep-ph/9311281]; L. J. Hall and H. Murayama, Phys. Rev. Lett. 75 (1995) 3985 arXiv:hep-ph/9508296]; C. D. Carone, L. J. Hall and H. Murayama, Phys. Rev. D 53 (1996) 6282 [arXiv:hep-ph/9512399].

[39] K. Hamaguchi, M. Kakizaki and M. Yamaguchi, Phys. Rev. D 68 (2003) 056007 arXiv:hep-ph/0212172 ; K. S. Babu, T. Kobayashi and J. Kubo, Phys. Rev. D 67 (2003) 075018 arXiv:hep-ph/0212350.

[40] T. Kobayashi, J. Kubo and H. Terao, Phys. Lett. B 568 (2003) 83 arXiv:hep-ph/0303084; S. F. King and G. G. Ross, Phys. Lett. B 574 (2003) 239 [arXiv:hep-ph/0307190].

[41] G. G. Ross, L. Velasco-Sevilla and O. Vives, Nucl. Phys. B 692 (2004) 50 arXiv:hep-ph/0401064.

[42] K. Y. Choi, Y. Kajiyama, H. M. Lee and J. Kubo, Phys. Rev. D 70 (2004) 055004 arXiv:hep-ph/0402026]; T. Kobayashi, S. Raby and R. J. Zhang, Nucl. Phys. B 704 (2005) 3 arXiv:hep-ph/0409098; M. Ciuchini, A. Masiero, P. Paradisi, L. Silvestrini, S. K. Vempati and O. Vives, Nucl. Phys. B 783 (2007) 112 arXiv:hep-ph/0702144]; M. C. Chen and K. T. Mahanthappa, Phys. Lett. B 652 (2007) 34 [arXiv:0705.0714 [hep-ph]]; I. de Medeiros Varzielas, S. F. King and G. G. Ross, Phys. Lett. B 648 (2007) 201 arXiv:hep-ph/0607045; I. de Medeiros Varzielas and G. G. Ross, arXiv:hep-ph/0612220; H. Ishimori, T. Kobayashi, H. Okada, Y. Shimizu and M. Tanimoto, JHEP 0912 (2009) 054 arXiv:0907.2006 [hep-ph]]; F. Feruglio, C. Hagedorn, Y. Lin and L. Merlo, Nucl. Phys. B 832 (2010) 251 arXiv:0911.3874 [hep-ph]]; L. Merlo, S. Rigolin and B. Zaldivar, JHEP 1111 (2011) 047 [arXiv:1108.1795 [hepph]]; L. Calibbi, J. Jones-Perez, A. Masiero, J. h. Park, W. Porod and O. Vives, Nucl. Phys. B 831 (2010) 26 [arXiv:0907.4069 [hep-ph]].

[43] N. Kifune, J. Kubo and A. Lenz, Phys. Rev. D 77 (2008) 076010 [arXiv:0712.0503 [hep-ph]]; K. Kawashima, J. Kubo and A. Lenz, Phys. Lett. B 681 (2009) 60 [arXiv:0907.2302 [hep-ph]].

[44] K. S. Babu and Y. Meng, Phys. Rev. D 80 (2009) 075003 [arXiv:0907.4231 [hep-ph]].

[45] J. Kubo and A. Lenz, Phys. Rev. D 82 (2010) 075001 [arXiv:1007.0680 [hep-ph]]; Y. Kaburaki, K. Konya, J. Kubo and A. Lenz, Phys. Rev. D 84 (2011) 016007 [arXiv:1012.2435 [hep-ph]].

[46] K. S. Babu, K. Kawashima and J. Kubo, Phys. Rev. D 83 (2011) 095008 arXiv:1103.1664 [hep-ph]].

[47] The Higgs sector of the minimal supersymmetri model (MSSM) is extensively reviewed in : A. Djouadi, Phys. Rept. 459 (2008) 1 arXiv:hep-ph/0503173, and see also J. Kubo, H. Okada and F. Sakamaki, Phys. Rev. D 70 (2004) 036007 arXiv:hep-ph/0402089 and R. de Adelhart Toorop, F. Bazzocchi, L. Merlo and A. Paris, JHEP 1103 (2011) 035 [arXiv:1012.1791] [hep$\mathrm{ph}]$.

[48] T. Kobayashi, J. Kubo and G. Zoupanos, Phys. Lett. B 427 (1998) 291 arXiv:hep-ph/9802267; I. Jack, D. R. T. Jones and A. Pickering, Phys. Lett. B 432 (1998) 114 arXiv:hep-ph/9803405. 
[49] J. C. Romao, Phys. Lett. B 173 (1986) 309; N. Maekawa, Phys. Lett. B 282 (1992) 387; J. R. Espinosa, J. M. Moreno and M. Quiros, Phys. Lett. B 319 (1993) 505 arXiv:hep-ph/9308315; K. S. Babu and S. M. Barr, Phys. Rev. D 49 (1994) 2156 arXiv:hep-ph/9308217; M. Masip and A. Rasin, Phys. Rev. D 52 (1995) 3768 arXiv:hep-ph/9506471]; Nucl. Phys. B 460 (1996) 449 arXiv:hep-ph/9508365; N. Haba, M. Matsuda and M. Tanimoto, Phys. Rev. D 54 (1996) 6928 [arXiv:hep-ph/9512421]; N. Haba, Phys. Lett. B 398 (1997) 305 arXiv:hep-ph/9609395]; M. Masip and A. Rasin, Phys. Rev. D 58 (1998) 035007 arXiv:hep-ph/9803271.

[50] Y. Okada, M. Yamaguchi and T. Yanagida, Prog. Theor. Phys. 85 (1991) 1; H. E. Haber and R. Hempfling, Phys. Rev. Lett. 66 (1991) 1815; J. R. Ellis, G. Ridolfi and F. Zwirner, Phys. Lett. B 257 (1991) 83.

[51] [ATLAS Collaboration], arXiv:1202.1408 [hep-ex].

[52] S. Chatrchyan et al. [CMS Collaboration], arXiv:1202.1488 [hep-ex].

[53] H. Fritzsch, Phys. Lett. B 73 (1978) 317; H. Fritzsch, Nucl. Phys. B 155 (1979) 189.

[54] G. C. Branco, L. Lavoura and F. Mota, Phys. Rev. D 39 (1989) 3443; D. s. Du and Z. z. Xing, Phys. Rev. D 48 (1993) 2349.

[55] H. Fritzsch and Z. z. Xing, Prog. Part. Nucl. Phys. 45 (2000) 1 arXiv:hep-ph/9912358; H. Fritzsch, Z. z. Xing and S. Zhou, JHEP 1109 (2011) 083 [arXiv:1108.4534 [hep-ph]].

[56] G. C. Branco, D. Emmanuel-Costa and C. Simoes, Phys. Lett. B 690 (2010) 62 arXiv:1001.5065 [hep-ph]]; D. Emmanuel-Costa and C. Simoes, Phys. Rev. D 85 (2012) 016003 [arXiv:1102.3729 [hep-ph]].

[57] Z. z. Xing, H. Zhang and S. Zhou, Phys. Rev. D 77 (2008) 113016. [arXiv:0712.1419 [hep-ph]].

[58] K. Nakamura et al. [Particle Data Group], J. Phys. G 37 (2010) 075021, and 2011 partial update for the 2012 edition.

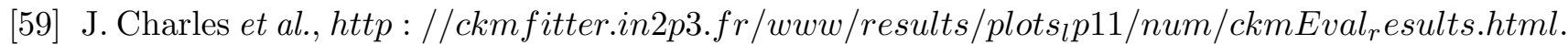

[60] A. Lenz et al., arXiv:1203.0238 [hep-ph].

[61] T. Schwetz, M. Tortola and J. W. F. Valle, New J. Phys. 13, 063004 (2011) arXiv:1103.0734 [hep-ph]].

[62] K. Abe et al. [T2K Collaboration], Phys. Rev. Lett. 107 (2011) 041801 arXiv:1106.2822 [hep-ex]].

[63] P. Adamson et al. [MINOS Collaboration], Phys. Rev. Lett. 107 (2011) 181802 arXiv:1108.0015 [hep-ex]].

[64] Y. Abe et al. [DOUBLE-CHOOZ Collaboration], arXiv:1112.6353 [hep-ex].

[65] F. P. An et al. [DAYA-BAY Collaboration], arXiv:1203.1669 [hep-ex].

[66] P. F. Harrison, D. H. Perkins and W. G. Scott, Phys. Lett. B 530 (2002) 167 arXiv:hep-ph/0202074; P. F. Harrison and W. G. Scott, Phys. Lett. B 535 (2002) 163 arXiv:hep-ph/0203209]; Z. z. Xing, Phys. Lett. B 533 (2002) 85 arXiv:hep-ph/0204049.

[67] F. Ardellier et al. [Double Chooz Collaboration], arXiv:hep-ex/0606025; X. Guo et al. [Daya-Bay Collaboration], arXiv:hep-ex/0701029; J. K. Ahn et al. [RENO Collaboration], arXiv:1003.1391 [hep-ex].

[68] S. R. Elliott, arXiv:1203.1070 [nucl-ex].

[69] A. Mondragon, M. Mondragon and E. Peinado, Phys. Rev. D 76 (2007) 076003 arXiv:0706.0354 [hep-ph]].

[70] S. Abel, S. Khalil and O. Lebedev, Nucl. Phys. B 606 (2001) 151 arXiv:hep-ph/0103320; M. Endo, M. Kakizaki and M. Yamaguchi, Phys. Lett. B 583 (2004) 186 arXiv:hep-ph/0311072; J. Hisano and Y. Shimizu, Phys. Rev. D 70 (2004) 093001 
arXiv:hep-ph/0406091; J. Hisano, M. Kakizaki, M. Nagai and Y. Shimizu, Phys. Lett. B 604 (2004) 216. arXiv:hep-ph/0407169].

[71] R. Barbieri, L. J. Hall and A. Strumia, Nucl. Phys. B 445 (1995) 219 arXiv:hep-ph/9501334; J. Hisano, T. Moroi, K. Tobe, M. Yamaguchi and T. Yanagida, Phys. Lett. B 357 (1995) 579 arXiv:hep-ph/9501407].

[72] F. Deppisch and J. W. F. Valle, Phys. Rev. D 72 (2005) 036001 arXiv:hep-ph/0406040; F. F. Deppisch, F. Plentinger and G. Seidl, JHEP 1101 (2011) 004 arXiv:1011.1404 [hep$\mathrm{ph}]]$.

[73] V. M. Abazov et al. [The D0 Collaboration], arXiv:1005.2757 [hep-ex]; V. M. Abazov et al. [The D0 Collaboration], arXiv:1007.0395 [hep-ex]; V. M. Abazov et al. [D0 Collaboration], Phys. Rev. D 84 (2011) 052007 [arXiv:1106.6308 [hep-ex]].

[74] T. Aaltonen et al. [CDF Collaboration], arXiv:1112.1726 [hep-ex].

[75] R. Aaij et al. [LHCb Collaboration], arXiv:1112.3183 [hep-ex].

[76] J. Adam et al. [MEG collaboration], Phys. Rev. Lett. 107 (2011) 171801 arXiv:1107.5547 [hep-ex]].

[77] K. Hayasaka, arXiv:1010.3746] [hep-ex].

[78] ATLAS Collaboration, arXiv:1203.0529 [hep-ex].

[79] S. Chatrchyan et al. [CMS Collaboration], Phys. Rev. Lett. 107 (2011) 221804 arXiv:1109.2352 [hep-ex]].

[80] G. Aad et al. [ATLAS Collaboration], arXiv:1109.6572 [hep-ex].

[81] B. Adeva et al. [The LHCb Collaboration], arXiv:0912.4179 [hep-ex].

[82] T. Aushev, W. Bartel, A. Bondar, J. Brodzicka, T. E. Browder, P. Chang, Y. Chao and K. F. Chen et al., arXiv:1002.5012 [hep-ex].

[83] G. Colangelo et al., Eur. Phys. J. C 71 (2011) 1695 arXiv:1011.4408 [hep-lat]].

[84] T. Araki and J. Kubo, Int. J. Mod. Phys. A 24 (2009) 5831 arXiv:0809.5136 [hep-ph]].

[85] L. Calibbi, R. N. Hodgkinson, J. Jones-Perez, A. Masiero and O. Vives, arXiv:1111.0176 [hepph]; S. S. AbdusSalam, B. C. Allanach, H. K. Dreiner, J. Ellis, U. Ellwanger, J. Gunion, S. Heinemeyer, M. Kraemer et al., arXiv:1109.3859 [hep-ph]. 